Noname manuscript No.

(will be inserted by the editor)

\title{
Optimal Control Problems with Stopping Constraints
}

\author{
Qun Lin · Ryan Loxton · Kok Lay Teo · Yong Hong \\ Wu
}

Received: date / Accepted: date

\begin{abstract}
We consider a novel optimal control problem in which the terminal time is governed by a stopping constraint. This stopping constraint is a nonlinear equality constraint depending on the state variables, and the terminal time is defined as the first time at which this constraint is satisfied. Since the stopping constraint causes the terminal time to be an implicit function of the control, the optimal control problem we consider cannot be solved using conventional techniques. We propose a new computational approach that involves approximating the original problem by a standard optimal control problem with fixed terminal time. Our main result shows that this approximation, which depends on two adjustable parameters, can be made to arbitrarily high accuracy. On this basis, the original optimal control problem with stopping constraints can be transformed into a sequence of approximate problems, each of which can be solved readily using conventional optimal control techniques. We conclude the paper by demonstrating this approach with numerical simulations in three application areas: range maximization of a hang glider, range maximization of a hypersonic re-entry vehicle, and time-optimal control of a nuclear reactor.
\end{abstract}

Keywords Nonlinear optimal control · Time-optimal control · Stopping constraint · Continuous inequality constraint · Time-scaling transformation

\section{Introduction}

The time-optimal control problem, a classic problem in control theory, involves determining a control law for manoeuvring a system from an initial state to a target state in minimum time. This problem is typically solved by considering the terminal time as a free decision variable and imposing a terminal state constraint to ensure that the system reaches the desired target at the terminal time $[9,16,20]$.

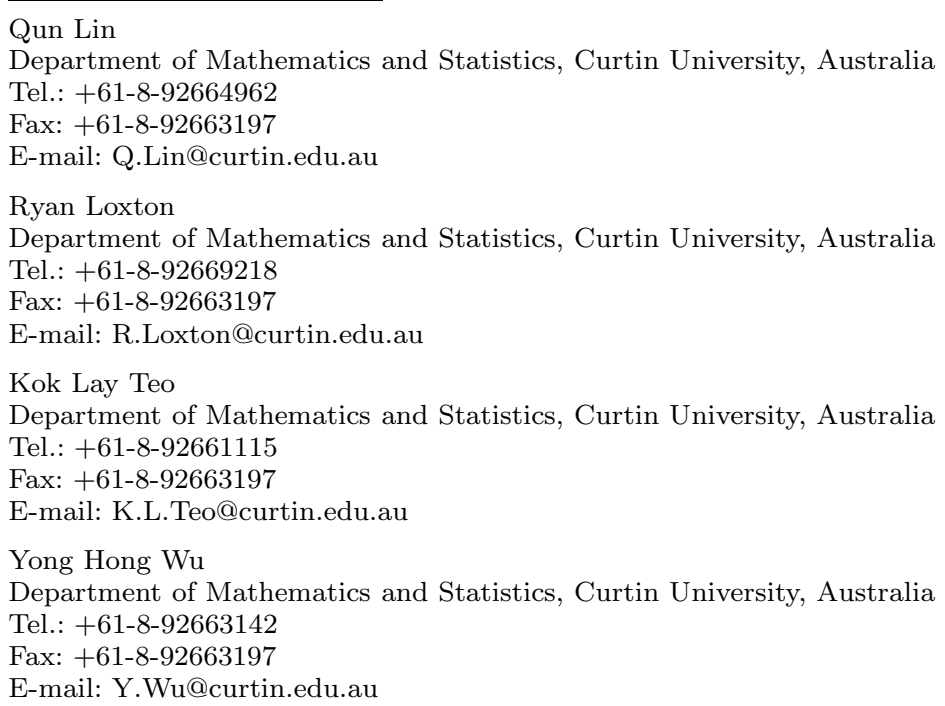


Since the terminal time is the quantity to be minimized, its optimal value will automatically be the first time at which the terminal state constraint is satisfied.

There are some optimal control problems, however, in which the terminal time is not necessarily the first time at which the terminal constraints are satisfied. An example is given in Section 6.1 of [12]. Traditional approaches for solving optimal control problems, such as the Pontryagin minimum principle [1] and numerical methods based on control parameterization [10,14, 20] and state discretization $[2,4,8]$, view terminal state constraints as boundary conditions rather than stopping constraints. Thus, these methods may generate solutions in which the terminal state constraints are satisfied at intermediate times, not just at the terminal time. This can lead to nonsensical results if the system under consideration is designed to cease operation once the terminal state constraints are satisfied.

For example, consider the glider control problem in Section 6.4 of [12]. In this problem, the state variables define the glider's position and velocity and the control variable is the glider's angle of attack. The aim is to vary the angle of attack during flight so that the glider's range is maximized. The dynamic equations describing the glider's motion are only valid when the glider's altitude is positive. Thus, the glider stops once its altitude reaches zero - a requirement that leads to a stopping constraint. If this constraint is imposed as a conventional terminal state constraint - that is, as a boundary constraint rather than a stopping constraint - then the dynamic model will admit invalid flight trajectories in which the glider's altitude becomes negative. In fact, we have observed that the "optimal" flight trajectory in this case does indeed reach negative altitudes. Thus, for the glider control problem to yield sensible results, the terminal time must be defined as the first time at which the glider's altitude is zero. Standard optimal control methods are not applicable in this case, as they cannot ensure that the glider's altitude is positive at all intermediate times.

The glider control problem described above differs considerably from standard optimal control problems in which the terminal time is fixed. The terminal time in the glider problem is actually an implicit function of the control - changing the glider's angle of attack changes its flight trajectory, which in turn changes the time at which the glider hits the ground. There are only a handful of references dealing with optimal control problems of this type, where the presence of stopping constraints causes the terminal time to be a function of the control. Such problems are discussed from a theoretical viewpoint in [3]. Regarding optimal control computation, there are several existing methods as discussed below.

The first computational method for solving optimal control problems with stopping constraints was developed in the late 1980s. This method, described in [21], was quickly superseded by another method in [19]. These early methods are based on a coarse discretization scheme in which the control is approximated by a piecewise-constant function with fixed knot points. This discretization scheme yields an approximate optimization problem that can be solved readily using nonlinear programming techniques, such as sequential quadratic programming [15,17]. Recently in [11], a superior piecewiseconstant discretization scheme was introduced in which both the control heights and the control knot points are decision variables to be chosen optimally. The cost of the suboptimal control generated by this discretization scheme converges to the true optimal cost as the discretization is refined. The method developed in [11] involves solving the approximate problem directly as a nonlinear programming problem. In [12], a new approach is proposed in which the approximate problem is solved indirectly by first transforming it into an equivalent problem that is easier to solve. Furthermore, in [6], the methods described in $[11,12]$ are extended to handle systems with continuous inequality constraints. These constraints arise in a wide range of important applications, such as hypersonic vehicle control [22] and isothermal semibatch reactors [23].

There is one major disadvantage shared by all of the methods mentioned above: they are based on the assumption that every admissible control transfers the system from the initial state to the stopping surface in finite time (the stopping surface is the surface defined by the stopping constraint). This assumption, which is essential for the methods in $[6,11,12,19,21]$ to be computationally feasible, is satisfied in the glider problem, but it is not a valid assumption for many other practical problems. In particular, for time-optimal control problems, the stopping surface consists of just a single point (the desired target state), and thus only some of the admissible controls will be capable of driving the system to the stopping surface. For this reason, the computational methods in $[6,11,12,19,21]$ are not effective at solving time-optimal control problems.

The purpose of this paper is to introduce a new computational method for solving optimal control problems with stopping constraints. This new method does not rely on the restrictive assumption that every admissible control transfers the system from the initial state to the stopping surface in 
finite time. Thus, it can be readily applied to solve time-optimal control problems. We illustrate this in Section 5.3, where we consider the time-optimal control of a nuclear reactor. Our new method can also solve more difficult problems such as the glider control problem described in [12]. Our approach involves approximating an optimal control problem with stopping constraints by a standard optimal control problem with conventional state constraints and fixed terminal time. This approximate problem can, in principle, be solved using the full gamut of traditional optimal control methods, such as state discretization, and control parameterization. Thus, unlike the previous methods in $[6,11,12,21]$, we are not restricted to any particular approximation scheme for the control. This is a key advantage, as we show in Section 5.1 that it is possible to generate improved results for the glider problem by using a piecewise-linear approximation scheme, instead of the piecewise-constant scheme used in $[6,11,12,21]$. A piecewise-linear approximation scheme also yields a more practical controller for this particular example.

The remainder of this paper is organized as follows. We first describe a general optimal control problem with stopping constraints in Section 2, before introducing a class of approximate problems in Section 3. We then prove our main convergence result in Section 4. In Section 5, we consider three numerical examples. Finally, we conclude the paper in Section 6.

\section{Problem Formulation}

\subsection{Nonlinear Control System}

We consider the following nonlinear control system:

$$
\dot{\boldsymbol{x}}(t)=\boldsymbol{f}(\boldsymbol{x}(t), \boldsymbol{u}(t)), \quad t \geq 0,
$$

and

$$
\boldsymbol{x}(0)=\boldsymbol{x}^{0}
$$

where $\boldsymbol{x}(t) \in \mathbb{R}^{n}$ is the state at time $t ; \boldsymbol{u}(t) \in \mathbb{R}^{r}$ is the control at time $t ; \boldsymbol{x}^{0} \in \mathbb{R}^{n}$ is a given initial state; and $\boldsymbol{f}: \mathbb{R}^{n} \times \mathbb{R}^{r} \rightarrow \mathbb{R}^{n}$ is a given continuously differentiable function.

Define

$$
\Omega \triangleq\left\{\omega \in \mathbb{R}^{r}: a_{i} \leq \omega_{i} \leq b_{i}, i=1, \ldots, r\right\},
$$

where $a_{i}$ and $b_{i}, i=1, \ldots, r$, are given real numbers such that $a_{i}<b_{i}$. Any Borel measurable function $\boldsymbol{u}:[0, \infty) \rightarrow \mathbb{R}^{r}$ such that $\boldsymbol{u}(t) \in \Omega$ for all $t \in[0, \infty)$ is called an admissible control. Let $\mathscr{U}$ denote the class of all such admissible controls.

As is customary in the optimal control literature (see $[1,11,12,14,20]$ ), we assume that there exists a real number $L>0$ such that

$$
\|\boldsymbol{f}(\boldsymbol{\zeta}, \boldsymbol{\omega})\| \leq L(1+\|\boldsymbol{\zeta}\|), \quad(\boldsymbol{\zeta}, \boldsymbol{\omega}) \in \mathbb{R}^{n} \times \Omega,
$$

where $\|\cdot\|$ denotes the Euclidean norm. This condition ensures that system (1)-(2) admits a unique Carathéodory solution for each admissible control $\boldsymbol{u} \in \mathscr{U}$ (see Theorem 3.1.6 of [1]). Let $\boldsymbol{x}(\cdot \mid \boldsymbol{u})$ denote this solution.

\subsection{Stopping Constraint}

Define the following stopping surface for system (1)-(2):

$$
\mathcal{X} \triangleq\left\{\zeta \in \mathbb{R}^{n}: \Phi(\boldsymbol{\zeta})=0\right\}
$$

where $\Phi: \mathbb{R}^{n} \rightarrow \mathbb{R}$ is a given continuously differentiable function. System (1)-(2) stops once its state trajectory reaches this surface. Thus, the system's terminal time is defined by

$$
T(\boldsymbol{u}) \triangleq \inf \{t>0: \boldsymbol{x}(t \mid \boldsymbol{u}) \in \mathcal{X}\}
$$

Note that the terminal time here is an implicit function of the control, as changing the control changes the state trajectory, which subsequently changes the time at which the state trajectory reaches the stopping surface. 
If $T(\boldsymbol{u})$ is infinite, then the set on the right-hand side of (4) is empty, and thus the control $\boldsymbol{u}$ fails to deliver the system to the stopping surface. On the other hand, if $T(\boldsymbol{u})$ is finite, then the continuity of $\Phi$ implies that

$$
\left.\Phi(\boldsymbol{x}(t \mid \boldsymbol{u}))\right|_{t=T(\boldsymbol{u})}=0 .
$$

Equation (5) can be viewed as a stopping constraint that defines the terminal time for (1)-(2). This is different from the usual terminal state constraints found in optimal control problems, which are allowed to be satisfied at intermediate times.

Throughout this paper, we assume that there exists a positive real number $T_{\min }>0$ such that $T(\boldsymbol{u}) \geq T_{\min }$ for each admissible control $\boldsymbol{u} \in \mathscr{U}$.

Clearly, $\Phi(\boldsymbol{x}(t \mid \boldsymbol{u})) \neq 0$ for all $t \in(0, T(\boldsymbol{u}))$. We assume without loss of generality that for each $\boldsymbol{u} \in \mathscr{U}$,

$$
\Phi(\boldsymbol{x}(t \mid \boldsymbol{u}))>0, \quad t \in(0, T(\boldsymbol{u})) .
$$

If this assumption is violated, then we can simply replace $\Phi$ with $\Phi^{2}$, since it is clear that $\Phi^{2}(\boldsymbol{x}(t \mid \boldsymbol{u}))>$ 0 for all $t \in(0, T(\boldsymbol{u}))$.

\subsection{The Optimal Control Problem}

Let $T_{\max }>T_{\min }$ be an upper bound for the terminal time. Then we have the following constraint:

$$
T(\boldsymbol{u}) \leq T_{\max }
$$

In addition to this constraint on the terminal time, we also have the following set of continuous inequality constraints on the state and control:

$$
h_{j}(\boldsymbol{x}(t \mid \boldsymbol{u}), \boldsymbol{u}(t)) \geq 0, \quad t \in[0, T(\boldsymbol{u})], \quad j=1, \ldots, m,
$$

where $h_{j}: \mathbb{R}^{n} \times \mathbb{R}^{r} \rightarrow \mathbb{R}, j=1, \ldots, m$, are given continuously differentiable functions.

Let $\mathscr{F}$ denote the set of all admissible controls $\boldsymbol{u} \in \mathscr{U}$ satisfying (7) and (8). Such controls are called feasible controls. We assume throughout this paper that $\mathscr{F} \neq \emptyset$.

Our optimal control problem is stated formally below.

Problem 1 Choose a feasible control $\boldsymbol{u} \in \mathscr{F}$ to minimize the cost function

$$
\left.G(\boldsymbol{u}) \triangleq \Psi(\boldsymbol{x}(t \mid \boldsymbol{u}))\right|_{t=T(\boldsymbol{u})},
$$

where $\Psi: \mathbb{R}^{n} \rightarrow \mathbb{R}$ is a given continuously differentiable function.

The main difficulty with Problem 1 is that the terminal constraint is only allowed to be satisfied at the terminal time, not at intermediate times. Because of this requirement, the terminal time in Problem 1 is actually a function of the control. Thus, standard optimal control methods, which are based on the assumption that the terminal time is fixed and known, are not applicable to Problem 1.

The glider control problem in [12] is a special case of Problem 1. In this problem, the stopping surface is the plane $h=0$, where $h$ is the glider's altitude (the glider stops once it hits the ground). The aim is to devise a control law for the glider so that the final range is maximized.

In addition to the glider problem mentioned above, the framework of Problem 1 subsumes the following important classes of optimal control problems:

(i) Optimal control problems with fixed terminal time (introduce a new state variable $x_{n+1}$ satisfying $\dot{x}_{n+1}(t)=1$ and $x_{n+1}(0)=0$, and put $\Phi(\boldsymbol{x})=x_{n+1}-t_{f}$, where $t_{f}$ is the given terminal time);

(ii) Time-optimal control problems (let $x_{n+1}$ be defined as above and put $\Psi(\boldsymbol{x})=x_{n+1}$ and $\Phi(\boldsymbol{x})=$ $\left\|\boldsymbol{x}-\boldsymbol{x}^{f}\right\|^{2}$, where $\boldsymbol{x}^{f}$ is the desired target state).

As we will see in Section 5, our new computational method for solving Problem 1 can effectively solve these standard problems, as well as more difficult problems such as the glider problem considered in $[12]$. 


\section{Problem Approximation}

In this section, we will show how to approximate Problem 1 by a standard optimal control problem with fixed terminal time. We first transform the time horizon in Problem 1 into the fixed interval $[0,1]$. This transformation removes the complication of having a variable terminal time, but it unfortunately results in a non-standard state constraint. Thus, we proceed by approximating the non-standard state constraint by a continuous inequality constraint depending on two adjustable parameters. This yields an approximate problem that can be solved using traditional optimal control methods.

\subsection{Transformation of the Time Horizon}

Let $s \in[0,1]$ be a new time variable. Furthermore, let $\mathscr{V}$ denote the set of all Borel measurable functions $\boldsymbol{v}:[0,1] \rightarrow \mathbb{R}^{r}$ such that $\boldsymbol{v}(s) \in \Omega$ for all $s \in[0,1]$, where $\Omega$ is as defined in Section 2 .

Consider the following dynamic system:

$$
\dot{\boldsymbol{y}}(s)=\gamma \boldsymbol{f}(\boldsymbol{y}(s), \boldsymbol{v}(s)), \quad s \in[0,1],
$$

and

$$
\boldsymbol{y}(0)=\boldsymbol{x}^{0}
$$

where $\boldsymbol{v} \in \mathscr{V}$ and $\gamma \in\left[T_{\min }, T_{\max }\right]$. Let $\boldsymbol{y}(\cdot \mid \boldsymbol{v}, \gamma):[0,1] \rightarrow \mathbb{R}^{n}$ denote the unique Carathéodory solution of (9)-(10).

By comparing the dynamic systems (1)-(2) and (9)-(10), the following result is immediately apparent.

Lemma 1 Let $(\boldsymbol{v}, \gamma) \in \mathscr{V} \times\left[T_{\min }, T_{\max }\right]$ and $\boldsymbol{u} \in \mathscr{U}$. Suppose that

$$
\boldsymbol{u}(t)=\boldsymbol{v}(t / \gamma), \quad t \in[0, \gamma]
$$

Then

$$
\boldsymbol{x}(t \mid \boldsymbol{u})=\boldsymbol{y}(t / \gamma \mid \boldsymbol{v}, \gamma), \quad t \in[0, \gamma]
$$

Define an extended real-valued function $S: \mathscr{V} \times\left[T_{\min }, T_{\max }\right] \rightarrow \mathbb{R} \cup\{\infty\}$ as follows:

$$
S(\boldsymbol{v}, \gamma) \triangleq \inf \{s \in(0,1]: \boldsymbol{y}(s \mid \boldsymbol{v}, \gamma) \in \mathcal{X}\}
$$

where $S(\boldsymbol{v}, \gamma)=\infty$ if the set on the right-hand side is empty. Furthermore, let $\mathscr{G}$ denote the set of all pairs $(\boldsymbol{v}, \gamma) \in \mathscr{V} \times\left[T_{\min }, T_{\max }\right]$ satisfying the following constraints:

$$
S(\boldsymbol{v}, \gamma)=1
$$

and

Clearly, for each $(\boldsymbol{v}, \gamma) \in \mathscr{G}$,

$$
h_{j}(\boldsymbol{y}(s \mid \boldsymbol{v}, \gamma), \boldsymbol{v}(s)) \geq 0, \quad s \in[0,1], \quad j=1, \ldots, m .
$$

$$
\Phi(\boldsymbol{y}(1 \mid \boldsymbol{v}, \gamma))=0
$$

where $\Phi$ is as defined in Section 2 .

Consider the following optimal control problem with fixed terminal time.

Problem 2 Choose a pair $(\boldsymbol{v}, \gamma) \in \mathscr{G}$ to minimize the cost function

$$
H(\boldsymbol{v}, \gamma) \triangleq \Psi(\boldsymbol{y}(1 \mid \boldsymbol{v}, \gamma))
$$

We will show that Problem 2 is equivalent to Problem 1 in the sense that a solution of either problem can be generated from a solution of the other. To do this, we need two preliminary lemmas, from which the main equivalence result follows easily.

Lemma 2 Let $(\boldsymbol{v}, \gamma) \in \mathscr{G}$ and define a corresponding function $\boldsymbol{u}:[0, \infty) \rightarrow \mathbb{R}^{r}$ as follows:

$$
\boldsymbol{u}(t)= \begin{cases}\boldsymbol{v}(t / \gamma), & \text { if } 0 \leq t \leq \gamma \\ \overline{\boldsymbol{\omega}}, & \text { if } t>\gamma,\end{cases}
$$

where $\overline{\boldsymbol{\omega}} \in \Omega$ is arbitrary. Then $\boldsymbol{u}$ is a feasible control for Problem 1. Furthermore, $T(\boldsymbol{u})=\gamma$ and $G(\boldsymbol{u})=H(\boldsymbol{v}, \gamma)$. 
Proof It is easy to see that $\boldsymbol{u} \in \mathscr{U}$. From Lemma 1 and equation (14),

$$
\Phi(\boldsymbol{x}(\gamma \mid \boldsymbol{u}))=\Phi(\boldsymbol{y}(1 \mid \boldsymbol{v}, \gamma))=0
$$

This implies that $T(\boldsymbol{u}) \leq \gamma$. By Lemma 1 and (5),

$$
\left.\Phi(\boldsymbol{y}(s \mid \boldsymbol{v}, \gamma))\right|_{s=T(\boldsymbol{u}) / \gamma}=\left.\Phi(\boldsymbol{x}(t \mid \boldsymbol{u}))\right|_{t=T(\boldsymbol{u})}=0 .
$$

Thus, since $S(\boldsymbol{v}, \gamma)=1$, we must have $T(\boldsymbol{u}) / \gamma=1$, which implies that $T(\boldsymbol{u})=\gamma$, as required.

We now show that $\boldsymbol{u} \in \mathscr{F}$. Clearly, since $\mathscr{G} \subset \mathscr{V} \times\left[T_{\min }, T_{\max }\right]$,

$$
T(\boldsymbol{u})=\gamma \leq T_{\max } .
$$

Furthermore, from Lemma 1 and inequality (13), for all $t \in[0, T(\boldsymbol{u})]=[0, \gamma]$,

$$
h_{j}(\boldsymbol{x}(t \mid \boldsymbol{u}), \boldsymbol{u}(t))=h_{j}(\boldsymbol{y}(t / \gamma \mid \boldsymbol{v}, \gamma), \boldsymbol{v}(t / \gamma)) \geq 0, \quad j=1, \ldots, m .
$$

Inequalities (16) and (17) show that $\boldsymbol{u} \in \mathscr{F}$.

Finally, by applying Lemma 1 once again, we obtain

$$
G(\boldsymbol{u})=\left.\Psi(\boldsymbol{x}(t \mid \boldsymbol{u}))\right|_{t=T(\boldsymbol{u})}=\Psi(\boldsymbol{x}(\gamma \mid \boldsymbol{u}))=\Psi(\boldsymbol{y}(1 \mid \boldsymbol{v}, \gamma))=H(\boldsymbol{v}, \gamma),
$$

as required.

Our second preliminary result is stated and proved below.

Lemma 3 Let $\boldsymbol{u} \in \mathscr{F}$ and define a corresponding function $\boldsymbol{v}:[0,1] \rightarrow \mathbb{R}^{r}$ as follows:

$$
\boldsymbol{v}(s)=\boldsymbol{u}(T(\boldsymbol{u}) s), \quad s \in[0,1] .
$$

Then $(\boldsymbol{v}, T(\boldsymbol{u})) \in \mathscr{G}$ and $H(\boldsymbol{v}, T(\boldsymbol{u}))=G(\boldsymbol{u})$.

Proof Since $\boldsymbol{u} \in \mathscr{F}$, it follows immediately that $T(\boldsymbol{u}) \in\left[T_{\min }, T_{\max }\right]$. Furthermore, it is clear that $\boldsymbol{v}$ defined by (18) belongs to $\mathscr{V}$. Thus, $(\boldsymbol{v}, T(\boldsymbol{u})) \in \mathscr{V} \times\left[T_{\min }, T_{\max }\right]$.

Note that equation (11) is satisfied for $\gamma=T(\boldsymbol{u})$. Thus, by Lemma 1,

$$
\boldsymbol{y}(s \mid \boldsymbol{v}, T(\boldsymbol{u}))=\boldsymbol{x}(T(\boldsymbol{u}) s \mid \boldsymbol{u}), \quad s \in[0,1] .
$$

Hence, from (5),

$$
\Phi(\boldsymbol{y}(1 \mid \boldsymbol{v}, T(\boldsymbol{u})))=\left.\Phi(\boldsymbol{x}(t \mid \boldsymbol{u}))\right|_{t=T(\boldsymbol{u})}=0 .
$$

Furthermore, from (6) and (19),

$$
\Phi(\boldsymbol{y}(s \mid \boldsymbol{v}, T(\boldsymbol{u})))=\left.\Phi(\boldsymbol{x}(t \mid \boldsymbol{u}))\right|_{t=T(\boldsymbol{u}) s}>0, \quad s \in(0,1) .
$$

In view of (20) and (21), we see that

$$
S(\boldsymbol{v}, T(\boldsymbol{u}))=1 .
$$

This proves equation (12). For the continuous inequality constraints (13), note that since $\boldsymbol{u} \in \mathscr{F}$,

$$
h_{j}(\boldsymbol{x}(t \mid \boldsymbol{u}), \boldsymbol{u}(t)) \geq 0, \quad t \in[0, T(\boldsymbol{u})], \quad j=1, \ldots, m .
$$

Hence, from (19),

$$
h_{j}(\boldsymbol{y}(s \mid \boldsymbol{v}, T(\boldsymbol{u})), \boldsymbol{v}(s))=\left.h_{j}(\boldsymbol{x}(t \mid \boldsymbol{u}), \boldsymbol{u}(t))\right|_{t=T(\boldsymbol{u}) s} \geq 0, \quad s \in[0,1], \quad j=1, \ldots, m .
$$

Together, (22) and (23) show that $(\boldsymbol{v}, T(\boldsymbol{u})) \in \mathscr{G}$.

Finally, by applying (19) again, we obtain

$$
H(\boldsymbol{v}, T(\boldsymbol{u}))=\Psi(\boldsymbol{y}(1 \mid \boldsymbol{v}, T(\boldsymbol{u})))=\left.\Psi(\boldsymbol{x}(t \mid \boldsymbol{u}))\right|_{t=T(\boldsymbol{u})}=G(\boldsymbol{u}) .
$$

This completes the proof.

Lemmas 2 and 3 show that each feasible pair in $\mathscr{G}$ has a corresponding feasible control in $\mathscr{F}$ with the same cost. On this basis, the following observations are apparent: 
1. Let $\left(\boldsymbol{v}^{*}, \gamma^{*}\right) \in \mathscr{G}$ be optimal for Problem 2 and define $\boldsymbol{u}^{*}:[0, \infty) \rightarrow \mathbb{R}^{r}$ as follows:

$$
\boldsymbol{u}^{*}(t)= \begin{cases}\boldsymbol{v}^{*}\left(t / \gamma^{*}\right), & \text { if } 0 \leq t \leq \gamma^{*} \\ \overline{\boldsymbol{\omega}}, & \text { if } t>\gamma^{*}\end{cases}
$$

where $\overline{\boldsymbol{\omega}} \in \Omega$ is arbitrary. Then $\boldsymbol{u}^{*}$ is optimal for Problem 1 .

2. Let $\boldsymbol{u}^{*} \in \mathscr{F}$ be optimal for Problem 1 and define $\boldsymbol{v}^{*}:[0,1] \rightarrow \mathbb{R}^{r}$ as follows:

$$
\boldsymbol{v}^{*}(s)=\boldsymbol{u}^{*}\left(T\left(\boldsymbol{u}^{*}\right) s\right), \quad s \in[0,1]
$$

Then $\left(\boldsymbol{v}^{*}, T\left(\boldsymbol{u}^{*}\right)\right)$ is optimal for Problem 2.

These two observations show that a solution of Problem 1 can be generated from a solution of Problem 2, and vice versa. Thus, we have established the following important result.

Theorem 1 (Equivalence Theorem) Problems 1 and 2 are equivalent.

\subsection{Constraint Approximation}

The "infimum" constraint $S(\boldsymbol{v}, \gamma)=1$ in Problem 2 is difficult to handle because $S(\boldsymbol{v}, \gamma)$ could be infinite. In previous work (see [12] and the references cited therein), this difficulty was overcome by assuming that $T(\boldsymbol{u})$ (and consequently $S(\boldsymbol{v}, \gamma)$ ) is always finite. However, as we explained in the introduction, this is a restrictive assumption that we would like to avoid.

In this paper, we take a different approach and replace $S(\boldsymbol{v}, \gamma)=1$ with the following two state constraints:

$$
\Phi(\boldsymbol{y}(1 \mid \boldsymbol{v}, \gamma))=0
$$

and

$$
\Phi(\boldsymbol{y}(s \mid \boldsymbol{v}, \gamma))>0, \quad s \in(0,1) .
$$

Clearly, $S(\boldsymbol{v}, \gamma)=1$ if and only if (24) and (25) are satisfied.

Constraint (25) is a non-standard "open" state constraint. We approximate this non-standard constraint by the following conventional constraint:

$$
\Phi(\boldsymbol{y}(s \mid \boldsymbol{v}, \gamma)) \geq \epsilon, \quad s \in[\delta, 1-\delta]
$$

where $\epsilon>0$ and $\delta \in\left(0, \frac{1}{2}\right)$ are adjustable parameters.

Let $\mathscr{G}_{\epsilon, \delta}$ denote the set of all pairs $(\boldsymbol{v}, \gamma) \in \mathscr{V} \times\left[T_{\min }, T_{\max }\right]$ satisfying (13), (24), and (26). Note that $S(\boldsymbol{v}, \gamma) \leq 1$ for each $(\boldsymbol{v}, \gamma) \in \mathscr{G}_{\epsilon, \delta}$.

We now consider the following approximation of Problem 2.

Problem 3 Choose a pair $(\boldsymbol{v}, \gamma) \in \mathscr{G}_{\epsilon, \delta}$ to minimize the cost function $H(\boldsymbol{v}, \gamma)$.

Problem 3 is a standard optimal control problem involving terminal state and continuous inequality constraints. Many well-known optimal control methods are available for solving such problems. In the next section, we will derive some convergence results linking Problem 3 with Problem 2.

\section{Convergence Results}

Since Problem 3 is an approximation of Problem 2, an obvious question to ask is: how good is this approximation? In this section, we will show that the approximation can be made to arbitrarily high accuracy by suitably adjusting the parameters $\epsilon$ and $\delta$. To do this, we require several preliminary lemmas. 
4.1 Preliminary Results

Suppose that $(\boldsymbol{v}, \gamma) \in \mathscr{V} \times\left[T_{\min }, T_{\max }\right]$ satisfies $S(\boldsymbol{v}, \gamma) \leq 1$. Let $\boldsymbol{u}$ be a corresponding function defined according to equation (15). It is easy to see that $\boldsymbol{u} \in \mathscr{\mathscr { U }}$ and $T(\boldsymbol{u}) \leq \gamma$. Using Lemma 1 , we have

$$
\boldsymbol{x}(t \mid \boldsymbol{u})=\boldsymbol{y}(t / \gamma \mid \boldsymbol{v}, \gamma), \quad t \in[0, \gamma]
$$

It thus follows from (6) that

$$
\Phi(\boldsymbol{y}(s \mid \boldsymbol{v}, \gamma))=\left.\Phi(\boldsymbol{x}(t \mid \boldsymbol{u}))\right|_{t=\gamma s}>0, \quad s \in(0, T(\boldsymbol{u}) / \gamma) .
$$

Furthermore, we know that

$$
\left.\Phi(\boldsymbol{y}(s \mid \boldsymbol{v}, \gamma))\right|_{s=T(\boldsymbol{u}) / \gamma}=\left.\Phi(\boldsymbol{x}(t \mid \boldsymbol{u}))\right|_{t=T(\boldsymbol{u})}=0 .
$$

Combining (27) and (28), we obtain

$$
S(\boldsymbol{v}, \gamma)=T(\boldsymbol{u}) / \gamma
$$

and

$$
\Phi(\boldsymbol{y}(s \mid \boldsymbol{v}, \gamma))>0, \quad s \in(0, S(\boldsymbol{v}, \gamma)) .
$$

These facts are used in the proofs of some of the following lemmas.

Lemma 4 For each $(\boldsymbol{v}, \gamma) \in \mathscr{G}$ and $\delta \in\left(0, \frac{1}{2}\right)$, there exists a corresponding real number $\bar{\epsilon} \triangleq$ $\bar{\epsilon}(\boldsymbol{v}, \gamma, \delta)>0$ such that $(\boldsymbol{v}, \gamma) \in \mathscr{G}_{\epsilon, \delta}$ whenever $\epsilon \in(0, \bar{\epsilon}]$.

Proof Let $(\boldsymbol{v}, \gamma) \in \mathscr{G}$ and $\delta \in\left(0, \frac{1}{2}\right)$. Then

$$
\Phi(\boldsymbol{y}(1 \mid \boldsymbol{v}, \gamma))=0
$$

and

Furthermore, by (30),

$$
h_{j}(\boldsymbol{y}(s \mid \boldsymbol{v}, \gamma), \boldsymbol{v}(s)) \geq 0, \quad s \in[0,1], \quad j=1, \ldots, m .
$$

$$
\Phi(\boldsymbol{y}(s \mid \boldsymbol{v}, \gamma))>0, \quad s \in(0,1) .
$$

Since $\Phi(\boldsymbol{y}(\cdot \mid \boldsymbol{v}, \gamma))$ is a continuous function of $s$, there exists an $s^{*} \triangleq s^{*}(\boldsymbol{v}, \gamma, \delta) \in[\delta, 1-\delta]$ such that

$$
\min _{s \in[\delta, 1-\delta]} \Phi(\boldsymbol{y}(s \mid \boldsymbol{v}, \gamma))=\Phi\left(\boldsymbol{y}\left(s^{*} \mid \boldsymbol{v}, \gamma\right)\right)>0
$$

where the last inequality follows from (33). Define $\bar{\epsilon} \triangleq \Phi\left(\boldsymbol{y}\left(s^{*} \mid \boldsymbol{v}, \gamma\right)\right)$ and let $\epsilon \in(0, \bar{\epsilon}]$ be arbitrary but fixed. Then

$$
\Phi(\boldsymbol{y}(s \mid \boldsymbol{v}, \gamma)) \geq \Phi\left(\boldsymbol{y}\left(s^{*} \mid \boldsymbol{v}, \gamma\right)\right)=\bar{\epsilon} \geq \epsilon, \quad s \in[\delta, 1-\delta]
$$

It follows from (31), (32), and (34) that $(\boldsymbol{v}, \gamma) \in \mathscr{G}_{\epsilon, \delta}$ whenever $\epsilon \in(0, \bar{\epsilon}]$.

Our next preliminary result shows that the state trajectory $\boldsymbol{y}(\cdot \mid \boldsymbol{v}, \gamma)$ is uniformly bounded on $[0,1]$ with respect to $(\boldsymbol{v}, \gamma)$.

Lemma 5 There exists a real number $C>0$ such that

$$
\max _{s \in[0,1]}\|\boldsymbol{y}(s \mid \boldsymbol{v}, \gamma)\| \leq C, \quad(\boldsymbol{v}, \gamma) \in \mathscr{V} \times\left[T_{\min }, T_{\max }\right]
$$

Proof Let $(\boldsymbol{v}, \gamma) \in \mathscr{V} \times\left[T_{\min }, T_{\max }\right]$ be arbitrary but fixed. From (9)-(10), we have

$$
\boldsymbol{y}(s \mid \boldsymbol{v}, \gamma)=\boldsymbol{x}^{0}+\int_{0}^{s} \gamma \boldsymbol{f}(\boldsymbol{y}(\tau \mid \boldsymbol{v}, \gamma), \boldsymbol{v}(\tau)) d \tau, \quad s \in[0,1]
$$

Thus, using (3),

$$
\begin{aligned}
\|\boldsymbol{y}(s \mid \boldsymbol{v}, \gamma)\| & \leq\left\|\boldsymbol{x}^{0}\right\|+T_{\max } L \int_{0}^{s}\{1+\|\boldsymbol{y}(\tau \mid \boldsymbol{v}, \gamma)\|\} d \tau \\
& \leq\left\|\boldsymbol{x}^{0}\right\|+T_{\max } L+T_{\max } L \int_{0}^{s}\|\boldsymbol{y}(\tau \mid \boldsymbol{v}, \gamma)\| d \tau .
\end{aligned}
$$

Applying Gronwall-Bellman's lemma gives

$$
\|\boldsymbol{y}(s \mid \boldsymbol{v}, \gamma)\| \leq L^{\prime} \exp \left(T_{\max } L s\right) \leq L^{\prime} \exp \left(T_{\max } L\right), \quad s \in[0,1],
$$

where $L^{\prime}=\left\|\boldsymbol{x}^{0}\right\|+T_{\max } L$. This completes the proof. 
Recall from Section 2 that $T(\boldsymbol{u}) \geq T_{\text {min }}$ for each admissible control $\boldsymbol{u} \in \mathscr{U}$. Based on this assumption and (29), we now prove that a similar property holds for $S(\boldsymbol{v}, \gamma)$.

Lemma 6 There exists a positive real number $S_{\min }>0$ such that

$$
S(\boldsymbol{v}, \gamma) \geq S_{\min }, \quad(\boldsymbol{v}, \gamma) \in \mathscr{V} \times\left[T_{\min }, T_{\max }\right] .
$$

Proof Suppose, to the contrary, that for each integer $k \in \mathbb{N}$, there exists a corresponding pair $\left(\boldsymbol{v}_{k}, \gamma_{k}\right) \in \mathscr{V} \times\left[T_{\min }, T_{\max }\right]$ such that

$$
S\left(\boldsymbol{v}_{k}, \gamma_{k}\right)<\frac{1}{k}
$$

Clearly, $S\left(\boldsymbol{v}_{k}, \gamma_{k}\right)<1$ for each $k \in \mathbb{N}$. Let $\boldsymbol{u}_{k}$ denote the admissible control in Lemma 2 corresponding to $\left(\boldsymbol{v}_{k}, \gamma_{k}\right)$. Then from $(29)$,

$$
T\left(\boldsymbol{u}_{k}\right)=\gamma_{k} S\left(\boldsymbol{v}_{k}, \gamma_{k}\right)<\frac{\gamma_{k}}{k} \leq \frac{T_{\max }}{k}, \quad k \geq 1
$$

Set $k^{\prime} \triangleq\left\lceil T_{\max } / T_{\min }\right\rceil$. Then it follows from the above inequality that for all integers $k \geq k^{\prime}$,

$$
T\left(\boldsymbol{u}_{k}\right)<\frac{T_{\max }}{k} \leq T_{\min }
$$

But this contradicts our assumption that $T(\boldsymbol{u}) \geq T_{\min }$ for each $\boldsymbol{u} \in \mathscr{U}$.

Our next result, which is based on Lemmas 5 and 6 , shows that the system cost at $s=S(\boldsymbol{v}, \gamma)$ is similar to the system cost at $s=1$ when $\delta$ is sufficiently small.

Lemma 7 Let $\varsigma>0$ be arbitrary but fixed. Then there exists a real number $\bar{\delta}>0$ such that for each $\delta \in(0, \bar{\delta})$ and $\epsilon>0$,

$$
|H(\boldsymbol{v}, \gamma)-\Psi(\boldsymbol{y}(s \mid \boldsymbol{v}, \gamma))|_{s=S(\boldsymbol{v}, \gamma)} \mid<\varsigma, \quad(\boldsymbol{v}, \gamma) \in \mathscr{G}_{\epsilon, \delta}
$$

Proof Since $\boldsymbol{y}(\cdot \mid \boldsymbol{v}, \gamma)$ and $\boldsymbol{v}$ are uniformly bounded on [0,1], and $\boldsymbol{f}$ and $\Psi$ are continuously differentiable, there exists a real number $C^{\prime}>0$ such that

$$
\left|\frac{\partial \Psi(\boldsymbol{y}(s \mid \boldsymbol{v}, \gamma))}{\partial \boldsymbol{x}} \boldsymbol{f}(\boldsymbol{y}(s \mid \boldsymbol{v}, \gamma), \boldsymbol{v}(s))\right| \leq C^{\prime}, \quad s \in[0,1], \quad(\boldsymbol{v}, \gamma) \in \mathscr{V} \times\left[T_{\min }, T_{\max }\right] .
$$

Let $s_{1}, s_{2} \in[0,1]$ be two fixed points. Then

$$
\Psi\left(\boldsymbol{y}\left(s_{2} \mid \boldsymbol{v}, \gamma\right)\right)-\Psi\left(\boldsymbol{y}\left(s_{1} \mid \boldsymbol{v}, \gamma\right)\right)=\gamma \int_{s_{1}}^{s_{2}} \frac{\partial \Psi(\boldsymbol{y}(s \mid \boldsymbol{v}, \gamma))}{\partial \boldsymbol{x}} \boldsymbol{f}(\boldsymbol{y}(s \mid \boldsymbol{v}, \gamma), \boldsymbol{v}(s)) d s
$$

Hence, from (35),

$$
\left|\Psi\left(\boldsymbol{y}\left(s_{2} \mid \boldsymbol{v}, \gamma\right)\right)-\Psi\left(\boldsymbol{y}\left(s_{1} \mid \boldsymbol{v}, \gamma\right)\right)\right| \leq \gamma C^{\prime}\left|s_{2}-s_{1}\right| \leq T_{\max } C^{\prime}\left|s_{2}-s_{1}\right| .
$$

This shows that $\Psi(\boldsymbol{y}(\cdot \mid \boldsymbol{v}, \gamma))$ is a Lipschitz function of $s$ on the interval $[0,1]$.

Now, define

$$
\bar{\delta} \triangleq \min \left\{S_{\min }, \frac{\varsigma}{T_{\max } C^{\prime}}, \frac{1}{2}\right\}
$$

where $S_{\text {min }}$ is as defined in Lemma 6 . Let $\delta \in(0, \bar{\delta})$ and $\epsilon>0$ be arbitrary but fixed. Then since $\delta<\bar{\delta} \leq S_{\min }$

$$
1-\delta<S(\boldsymbol{v}, \gamma) \leq 1, \quad(\boldsymbol{v}, \gamma) \in \mathscr{G}_{\epsilon, \delta}
$$

Thus,

$$
|1-S(\boldsymbol{v}, \gamma)|=1-S(\boldsymbol{v}, \gamma)<\delta
$$

Using (36) and the Lipschitz property of $\Psi(\boldsymbol{y}(\cdot \mid \boldsymbol{v}, \gamma))$, we have

$$
\begin{aligned}
|H(\boldsymbol{v}, \gamma)-\Psi(\boldsymbol{y}(s \mid \boldsymbol{v}, \gamma))|_{s=S(\boldsymbol{v}, \gamma)} \mid & =|\Psi(\boldsymbol{y}(1 \mid \boldsymbol{v}, \gamma))-\Psi(\boldsymbol{y}(s \mid \boldsymbol{v}, \gamma))|_{s=S(\boldsymbol{v}, \gamma)} \mid \\
& \leq T_{\max } C^{\prime}|1-S(\boldsymbol{v}, \gamma)|<T_{\max } C^{\prime} \delta<T_{\max } C^{\prime} \bar{\delta} \leq \varsigma
\end{aligned}
$$

This completes the proof. 
Our final preliminary result shows that any pair in $\mathscr{G}_{\epsilon, \delta}$ can be transformed into an "equivalent" pair in $\mathscr{G}$

Lemma 8 Suppose that $(\boldsymbol{v}, \gamma) \in \mathscr{V} \times\left[T_{\min }, T_{\max }\right]$ satisfies the continuous inequality constraints (13) and the terminal constraint (24). Furthermore, define $\overline{\boldsymbol{v}}:[0,1] \rightarrow \mathbb{R}^{r}$ as follows:

$$
\overline{\boldsymbol{v}}(s)=\boldsymbol{v}(S(\boldsymbol{v}, \gamma) s), \quad s \in[0,1] .
$$

Then $(\overline{\boldsymbol{v}}, \gamma S(\boldsymbol{v}, \gamma)) \in \mathscr{G}$ and

$$
H(\overline{\boldsymbol{v}}, \gamma S(\boldsymbol{v}, \gamma))=\left.\Psi(\boldsymbol{y}(s \mid \boldsymbol{v}, \gamma))\right|_{s=S(\boldsymbol{v}, \gamma)} \cdot
$$

Proof We first show that $(\overline{\boldsymbol{v}}, \gamma S(\boldsymbol{v}, \gamma)) \in \mathscr{V} \times\left[T_{\min }, T_{\max }\right]$. Since $(\boldsymbol{v}, \gamma)$ satisfies $(24)$, we have $S(\boldsymbol{v}, \gamma) \leq$ 1 , and thus $\overline{\boldsymbol{v}}$ in (37) is well-defined and belongs in $\mathscr{V}$. Furthermore,

$$
\gamma S(\boldsymbol{v}, \gamma) \leq \gamma \leq T_{\max }
$$

Let $\boldsymbol{u}$ denote the admissible control in Lemma 2 corresponding to $(\boldsymbol{v}, \gamma)$. Then, by (29),

$$
\gamma S(\boldsymbol{v}, \gamma)=T(\boldsymbol{u}) \geq T_{\min }
$$

It follows from inequalities (38) and (39) that $\gamma S(\boldsymbol{v}, \gamma) \in\left[T_{\min }, T_{\max }\right]$. Thus, we have shown that $(\overline{\boldsymbol{v}}, \gamma S(\boldsymbol{v}, \gamma)) \in \mathscr{V} \times\left[T_{\min }, T_{\max }\right]$, as required.

Now, by viewing the dynamic system (9)-(10), it is easy to see that

$$
\boldsymbol{y}(s \mid \overline{\boldsymbol{v}}, \gamma S(\boldsymbol{v}, \gamma))=\left.\boldsymbol{y}(\tau \mid \boldsymbol{v}, \gamma)\right|_{\tau=S(\boldsymbol{v}, \gamma) s}, \quad s \in[0,1]
$$

Thus,

$$
\Phi(\boldsymbol{y}(1 \mid \overline{\boldsymbol{v}}, \gamma S(\boldsymbol{v}, \gamma)))=\Phi(\boldsymbol{y}(S(\boldsymbol{v}, \gamma) \mid \boldsymbol{v}, \gamma))=0
$$

and

$$
\Phi(\boldsymbol{y}(s \mid \overline{\boldsymbol{v}}, \gamma S(\boldsymbol{v}, \gamma)))=\left.\Phi(\boldsymbol{y}(\tau \mid \boldsymbol{v}, \gamma))\right|_{\tau=S(\boldsymbol{v}, \gamma) s}>0, \quad s \in(0,1)
$$

The second inequality follows from $(30)$ since $S(\boldsymbol{v}, \gamma) \leq 1$. Hence, $S(\overline{\boldsymbol{v}}, \gamma S(\boldsymbol{v}, \gamma))=1$.

Recall that $(\boldsymbol{v}, \gamma)$ satisfies the continuous inequality constraints (13). Thus, from (40),

$$
h_{j}(\boldsymbol{y}(s \mid \overline{\boldsymbol{v}}, \gamma S(\boldsymbol{v}, \gamma)), \overline{\boldsymbol{v}}(s))=\left.h_{j}(\boldsymbol{y}(\tau \mid \boldsymbol{v}, \gamma), \boldsymbol{v}(\tau))\right|_{\tau=S(\boldsymbol{v}, \gamma) s} \geq 0, \quad s \in[0,1], \quad j=1, \ldots, m
$$

It follows that $(\overline{\boldsymbol{v}}, \gamma S(\boldsymbol{v}, \gamma)) \in \mathscr{G}$.

Finally, invoking (40) once more yields

$$
H(\overline{\boldsymbol{v}}, \gamma S(\boldsymbol{v}, \gamma))=\Psi(\boldsymbol{y}(1 \mid \overline{\boldsymbol{v}}, \gamma S(\boldsymbol{v}, \gamma)))=\left.\Psi(\boldsymbol{y}(s \mid \boldsymbol{v}, \gamma))\right|_{s=S(\boldsymbol{v}, \gamma)},
$$

which completes the proof.

\subsection{The Main Convergence Result}

Suppose that we solve Problem 3 to obtain an optimal solution $\left(\boldsymbol{v}_{\epsilon, \delta}^{*}, \gamma_{\epsilon, \delta}^{*}\right)$. Then $\left(\boldsymbol{v}_{\epsilon, \delta}^{*}, \gamma_{\epsilon, \delta}^{*}\right)$ may or may not be feasible for Problem 2. In the latter case, we can use the procedure in Lemma 8 to generate a new pair $\left(\overline{\boldsymbol{v}}_{\epsilon, \delta}^{*}, \gamma_{\epsilon, \delta}^{*} S\left(\boldsymbol{v}_{\epsilon, \delta}^{*}, \gamma_{\epsilon, \delta}^{*}\right)\right)$ that is feasible for Problem 2. Our main convergence result, which we now prove, shows that the cost of $\left(\overline{\boldsymbol{v}}_{\epsilon, \delta}^{*}, \gamma_{\epsilon, \delta}^{*} S\left(\boldsymbol{v}_{\epsilon, \delta}^{*}, \gamma_{\epsilon, \delta}^{*}\right)\right) \in \mathscr{G}$ approaches the optimal cost of Problem 2 when $\epsilon$ and $\delta$ approach zero. On this basis, $\left(\overline{\boldsymbol{v}}_{\epsilon, \delta}^{*}, \gamma_{\epsilon, \delta}^{*} S\left(\boldsymbol{v}_{\epsilon, \delta}^{*}, \gamma_{\epsilon, \delta}^{*}\right)\right)$ is a good approximation of the solution of Problem 2 when $\epsilon$ and $\delta$ are small.

Theorem 2 Let $\left(\boldsymbol{v}^{*}, \gamma^{*}\right)$ and $\left(\boldsymbol{v}_{\epsilon, \delta}^{*}, \gamma_{\epsilon, \delta}^{*}\right)$ denote optimal solutions of Problems 2 and 3, respectively. Furthermore, let $\varsigma>0$ be arbitrary but fixed. Then for all sufficiently small $\delta>0$, there exists a corresponding real number $\bar{\epsilon} \triangleq \bar{\epsilon}(\delta)>0$ such that

$$
\left|H\left(\boldsymbol{v}^{*}, \gamma^{*}\right)-H\left(\overline{\boldsymbol{v}}_{\epsilon, \delta}^{*}, \gamma_{\epsilon, \delta}^{*} S\left(\boldsymbol{v}_{\epsilon, \delta}^{*}, \gamma_{\epsilon, \delta}^{*}\right)\right)\right|<\varsigma, \quad \epsilon \in(0, \bar{\epsilon}]
$$

where $\overline{\boldsymbol{v}}_{\epsilon, \delta}^{*}$ is defined in terms of $\left(\boldsymbol{v}_{\epsilon, \delta}^{*}, \gamma_{\epsilon, \delta}^{*}\right)$ according to equation (37). 
Proof It follows from Lemma 7 that there exists a real number $\bar{\delta}>0$ such that for each $\delta \in(0, \bar{\delta})$ and $\epsilon>0$,

$$
|H(\boldsymbol{v}, \gamma)-\Psi(\boldsymbol{y}(s \mid \boldsymbol{v}, \gamma))|_{s=S(\boldsymbol{v}, \gamma)} \mid<\varsigma, \quad(\boldsymbol{v}, \gamma) \in \mathscr{G}_{\epsilon, \delta}
$$

Let $\delta \in(0, \bar{\delta})$ be arbitrary but fixed. Using Lemma 4, there exists a corresponding real number $\bar{\epsilon}>0$ such that $\left(\boldsymbol{v}^{*}, \gamma^{*}\right) \in \mathscr{G}_{\epsilon, \delta}$ whenever $\epsilon \in(0, \bar{\epsilon}]$. Hence, for each $\epsilon \in(0, \bar{\epsilon}]$,

$$
H\left(\boldsymbol{v}_{\epsilon, \delta}^{*}, \gamma_{\epsilon, \delta}^{*}\right) \leq H\left(\boldsymbol{v}^{*}, \gamma^{*}\right)
$$

By Lemma 8,

$$
H\left(\overline{\boldsymbol{v}}_{\epsilon, \delta}^{*}, \gamma_{\epsilon, \delta}^{*} S\left(\boldsymbol{v}_{\epsilon, \delta}^{*}, \gamma_{\epsilon, \delta}^{*}\right)\right)=\left.\Psi\left(\boldsymbol{y}\left(s \mid \boldsymbol{v}_{\epsilon, \delta}^{*}, \gamma_{\epsilon, \delta}^{*}\right)\right)\right|_{s=S\left(\boldsymbol{v}_{\epsilon, \delta}^{*}, \gamma_{\epsilon, \delta}^{*}\right)} .
$$

Hence, for each $\epsilon \in(0, \bar{\epsilon}]$,

$$
\begin{aligned}
\mid H\left(\boldsymbol{v}^{*}, \gamma^{*}\right)-H\left(\overline{\boldsymbol{v}}_{\epsilon, \delta}^{*}, \gamma_{\epsilon, \delta}^{*}\right. & \left.S\left(\boldsymbol{v}_{\epsilon, \delta}^{*}, \gamma_{\epsilon, \delta}^{*}\right)\right) \mid \\
& =H\left(\overline{\boldsymbol{v}}_{\epsilon, \delta}^{*}, \gamma_{\epsilon, \delta}^{*} S\left(\boldsymbol{v}_{\epsilon, \delta}^{*}, \gamma_{\epsilon, \delta}^{*}\right)\right)-H\left(\boldsymbol{v}^{*}, \gamma^{*}\right) \\
& =\left.\Psi\left(\boldsymbol{y}\left(s \mid \boldsymbol{v}_{\epsilon, \delta}^{*}, \gamma_{\epsilon, \delta}^{*}\right)\right)\right|_{s=S\left(\boldsymbol{v}_{\epsilon, \delta}^{*}, \gamma_{\epsilon, \delta}^{*}\right)}-H\left(\boldsymbol{v}^{*}, \gamma^{*}\right) \\
& \leq\left.\Psi\left(\boldsymbol{y}\left(s \mid \boldsymbol{v}_{\epsilon, \delta}^{*}, \gamma_{\epsilon, \delta}^{*}\right)\right)\right|_{s=S\left(\boldsymbol{v}_{\epsilon, \delta}^{*}, \gamma_{\epsilon, \delta}^{*}\right)}-H\left(\boldsymbol{v}_{\epsilon, \delta}^{*}, \gamma_{\epsilon, \delta}^{*}\right) .
\end{aligned}
$$

Therefore, from (41),

$$
\begin{aligned}
\mid H\left(\boldsymbol{v}^{*}, \gamma^{*}\right) & -H\left(\overline{\boldsymbol{v}}_{\epsilon, \delta}^{*}, \gamma_{\epsilon, \delta}^{*} S\left(\boldsymbol{v}_{\epsilon, \delta}^{*}, \gamma_{\epsilon, \delta}^{*}\right)\right) \mid \\
& \leq\left|H\left(\boldsymbol{v}_{\epsilon, \delta}^{*}, \gamma_{\epsilon, \delta}^{*}\right)-\Psi\left(\boldsymbol{y}\left(s \mid \boldsymbol{v}_{\epsilon, \delta}^{*}, \gamma_{\epsilon, \delta}^{*}\right)\right)\right|_{s=S\left(\boldsymbol{v}_{\epsilon, \delta}^{*}, \gamma_{\epsilon, \delta}^{*}\right)} \mid<\varsigma .
\end{aligned}
$$

Since $\delta \in(0, \bar{\delta})$ was chosen arbitrarily and the above inequality holds for all $\epsilon \in(0, \bar{\epsilon}]$, the proof is complete.

We now conclude this section by proposing a computational strategy for solving Problem 1 . The main idea of this strategy is to solve Problem 3 for a decreasing sequence of $\epsilon$ and $\delta$, where the optimal solution at each stage is used as the initial guess for the next. At the final stage, when $\epsilon$ and $\delta$ are sufficiently small, the optimal solution of Problem 3 is transformed into an equivalent feasible pair for Problem 2 using the procedure in Lemma 8. According to Theorem 2, this equivalent pair is a good approximation of the solution of Problem 2. A corresponding suboptimal control for Problem 1 can then be generated via equation (15). Thus, we have essentially transformed Problem 1, an optimal control problem with stopping constraints, into a sequence of conventional optimal control problems.

Recall that Problem 3 is a nonlinear optimal control problem subject to terminal state and continuous inequality constraints. Although analytical techniques for solving such problems are available (see [5]), in practice, Problem 3 will usually be too complex to solve analytically. In the next section, we solve several example problems by using a combination of the control parameterization method [20], the time-scaling transformation [10], and a recently-developed exact penalty method [13].

\section{Numerical Examples}

We now consider three example problems: the range maximization of a hang glider, the range maximization of a hypersonic re-entry vehicle, and the time-optimal control of a nuclear reactor. To solve these problems, we wrote a Fortran program that implements the approximation approach described in Section 3. This program solves Problem 3, the approximate problem, using the control parameterization method (to discretize the control space), the time-scaling transformation (to optimize the discretization knot points), and the exact penalty method (to handle the continuous inequality constraints). Full details of the discretization procedure are given in [10]. Our program uses the optimization code NLPQLP [18] (which is based on the sequential quadratic programming method) to solve the discretized nonlinear programming problem obtained via control parameterization, and the Runge-Kutta method of order 6 to solve the differential equations. 


\subsection{Range Maximization of a Hang Glider}

We consider the glider control problem in $[11,12,19,21]$. The dynamics in this problem are given by the following differential equations:

$$
\begin{aligned}
& \dot{x}_{1}=x_{3} \cos \left(x_{4}\right), \\
& \dot{x}_{2}=x_{3} \sin \left(x_{4}\right), \\
& \dot{x}_{3}=-\left(k_{1}+k_{2} u^{2}\right) x_{3}^{2}-g \sin \left(x_{4}\right), \\
& \dot{x}_{4}=k_{3} x_{3} u-\frac{g \cos \left(x_{4}\right)}{x_{3}}
\end{aligned}
$$

and

$$
x_{1}(0)=0, \quad x_{2}(0)=0, \quad x_{3}(0)=370, \quad x_{4}(0)=\frac{3}{2},
$$

where $x_{1}$ is the glider's horizontal position (metres); $x_{2}$ is the glider's altitude (metres); $x_{3}$ is the glider's speed (metres per second); $x_{4}$ is the angle between the glider's velocity vector and the horizon (radians); $u$ is the glider's angle of attack (radians); $g=9.8 \mathrm{~ms}^{-2}$ is the gravitational acceleration; and $k_{1}=3.289 \times 10^{-5} \mathrm{~m}^{-1}, k_{2}=1.133 \times 10^{-3} \mathrm{~m}^{-1}$, and $k_{3}=3.289 \times 10^{-3} \mathrm{~m}^{-1}$ are fixed constants.

The glider is controlled by varying its angle of attack. The aim is to manipulate the angle of attack so that the glider flies as far as possible before crashing. The crash time $T$ is defined as the first time at which the following stopping constraint is satisfied:

$$
x_{2}(T)=0 .
$$

Our optimal control problem is defined as follows: Choose the angle of attack u to maximize $x_{1}(T)$ subject to the dynamics (42)-(43) and the stopping constraint (44).

We first attempt to solve this problem using the standard methodology for free terminal time optimal control problems (i.e. impose (44) as a boundary condition rather than a stopping constraint). As we will see, this standard methodology produces invalid solutions in which the glider's altitude becomes negative.

By applying the well-known time transformation $s=t / T$, the dynamic equations (42)-(43) are transformed into the following system defined on $[0,1]$ :

$$
\begin{aligned}
& \dot{x}_{1}=T x_{3} \cos \left(x_{4}\right), \\
& \dot{x}_{2}=T x_{3} \sin \left(x_{4}\right), \\
& \dot{x}_{3}=-T\left(k_{1}+k_{2} u^{2}\right) x_{3}^{2}-g T \sin \left(x_{4}\right), \\
& \dot{x}_{4}=k_{3} T x_{3} u-\frac{g T \cos \left(x_{4}\right)}{x_{3}}
\end{aligned}
$$

and

$$
x_{1}(0)=0, \quad x_{2}(0)=0, \quad x_{3}(0)=370, \quad x_{4}(0)=\frac{3}{2},
$$

where $s \in[0,1]$ is the new time variable and $T$ is now a decision variable. The stopping constraint (44) is transformed into the following terminal state constraint:

$$
x_{2}(1)=0 .
$$

The new optimal control problem is defined as follows: Choose $T$ and $u$ to maximize $x_{1}(1)$ subject to the dynamics (45)-(46) and the terminal state constraint (47).

This is a standard optimal control problem with fixed terminal time. Using the control parameterization method with $p=3$, we solved this problem to yield an optimal terminal time of $T=386.08$ with corresponding maximum range $x_{1}(T)=48,298.84$. The optimal flight trajectory is shown in Figure 1. Note that the glider's altitude becomes negative near the terminal time. Thus, the optimal solution shown here is invalid. This indicates that the standard methodology for solving free terminal time optimal control problems (i.e. consider the stopping constraint as a terminal state constraint) is inadequate for the glider control problem.

We now use our new computational method to solve the glider control problem. We consider two approximation schemes for the control: piecewise-constant control parameterization and piecewiselinear control parameterization. In the piecewise-linear case, the angle of attack $u$ is viewed as an additional state variable with dynamics

$$
\dot{u}(t)=v(t), \quad u(0)=u_{0},
$$




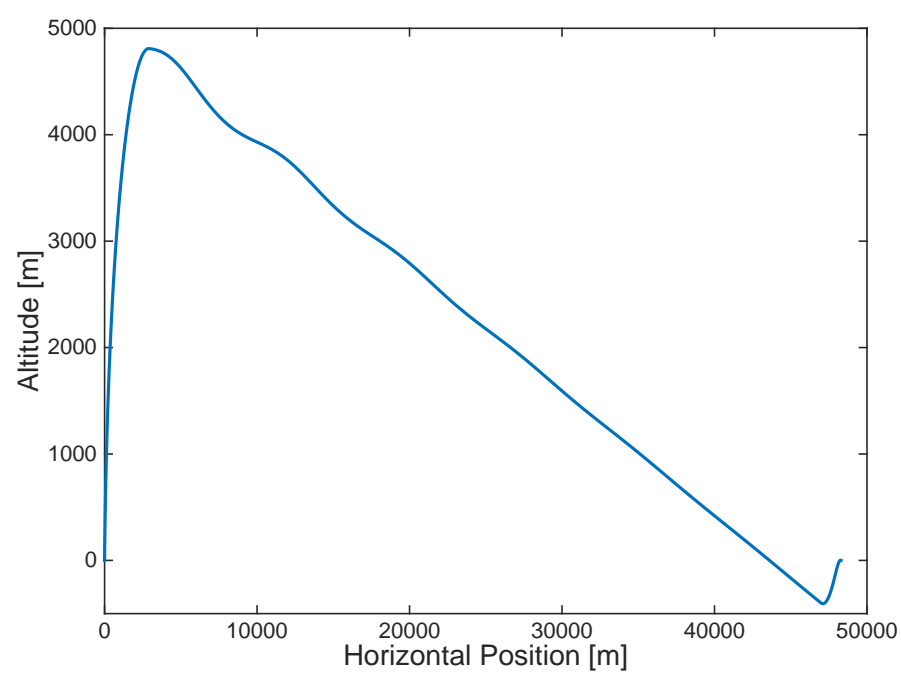

(a) Optimal flight trajectory

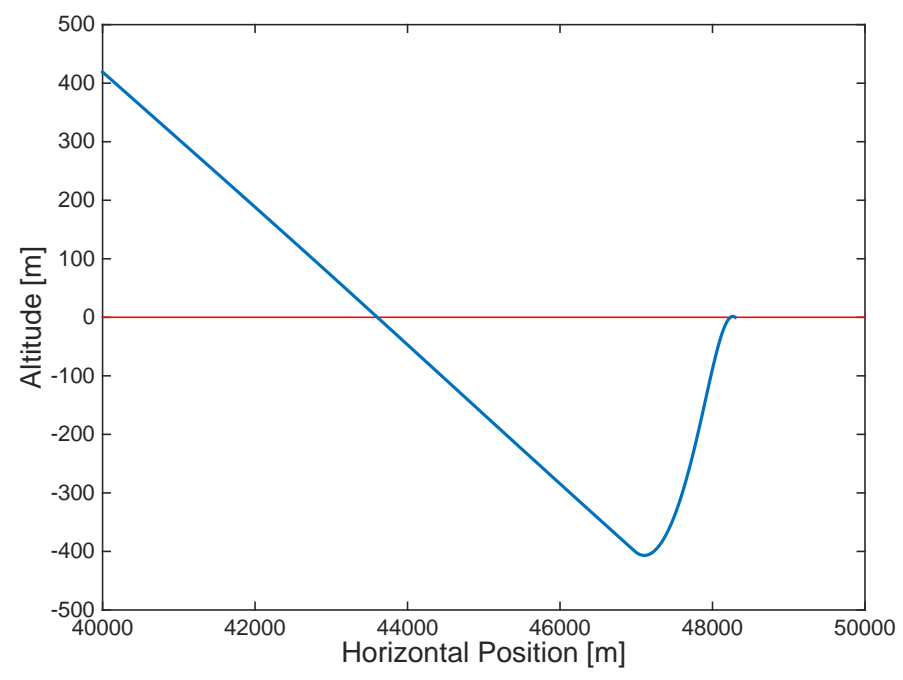

(b) Optimal flight trajectory near the terminal time

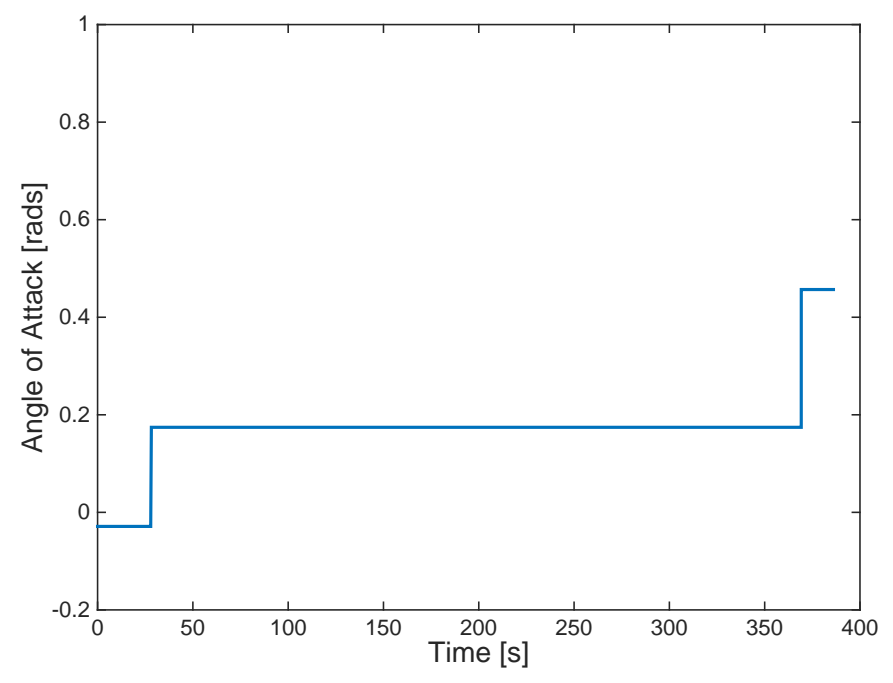

(c) Optimal angle of attack function

Fig. 1: Optimal solution of the transformed glider control problem in Section 5.1 (all functions are plotted with respect to the original time variable $t$ ). Note that the glider's altitude becomes negative near the terminal time. 


\begin{tabular}{|c|c|c|c|c|c|c|c|c|c|}
\hline$p$ & \multicolumn{3}{|c|}{ Piecewise-constant } & \multicolumn{3}{|c|}{ Piecewise-linear } & \multicolumn{3}{|c|}{ Method in [12] } \\
\hline 2 & 370.22 & 44038.90 & 3 & 362.59 & 42692.72 & 35 & 370.32 & 44038.82 & 20 \\
\hline 3 & 383.12 & 46568.91 & 7 & 369.36 & 45155.20 & 7 & 386.87 & 46512.53 & 54 \\
\hline 4 & 388.84 & 47717.99 & 7 & 387.39 & 47531.14 & 14 & 379.51 & 47274.39 & 3 \\
\hline 7 & 390.34 & 48320.09 & 39 & 391.63 & 48614.55 & 59 & & & \\
\hline
\end{tabular}

Table 1: Numerical results for the glider control problem in Section 5.1.

\begin{tabular}{cccccc}
\hline & \multicolumn{2}{c}{ Piecewise-constant } & & \multicolumn{2}{c}{ Piecewise-linear } \\
\cline { 2 - 3 } \cline { 5 - 6 }$p$ & $T$ & $x_{1}(T)$ & & $T$ & $x_{1}(T)$ \\
\hline 2 & 370.22 & 44038.90 & & 336.00 & 42135.24 \\
3 & 341.94 & 45383.77 & & 351.58 & 44607.29 \\
4 & 341.47 & 45518.00 & & 343.09 & 45011.71 \\
5 & 352.43 & 45700.74 & & 350.42 & 45784.40 \\
6 & 352.31 & 45742.63 & & 351.77 & 45811.86 \\
7 & 356.36 & 45803.25 & & 354.03 & 45853.85 \\
\hline
\end{tabular}

Table 2: Numerical results for the glider control problem in Section 5.1 with control constraints (49).

where $v$ is a new piecewise-constant control function and $u_{0}$ is a fixed constant (in the results that follow, $\left.u_{0}=-0.048\right)$. By appending (48) to the original dynamic system, the piecewise-constant control parameterization method can be applied to the piecewise-linear case. See [10] for more details.

We choose $\epsilon=10$ and $\delta=10^{-2}$. Note that we have deliberately chosen a large value of $\epsilon$ to avoid flight trajectories that "skim" the ground near the terminal time (the results in [12] indicate that the optimal trajectory comes to within 1 metre of the ground at an intermediate time before re-launching into a steep climb). By choosing $\epsilon=10$, the control will try and keep the glider at least 10 metres above the ground for as long as possible.

The optimal angle of attack functions and corresponding flight trajectories are shown in Figure 2 and Figure 3. The results shown are consistent with those reported in $[11,12,19,21]$. Note that the piecewise-linear approximation yields better results than the piecewise-constant approximation used in $[11,12,21]$.

For comparison, we applied the numerical method developed in [12] to the glider problem with stopping constraint $x_{2}(T)=10$. The optimal results are shown in Table 1 and Figure 4 . Our experience is that this method only works if the cost function's gradient is normalized, as the terminal time is free and very sensitive to changes in the decision variables. The results given in Table 1 are the best results we could find using this method. After $p=6$, there was no improvement in the optimal range.

The optimal controls in Figure 2 and Figure 3 are difficult to implement in a real glider because the angle of attack is extremely steep at the end of the time horizon. Thus, we improve the model by imposing the following bound constraint on the angle of attack:

$$
-0.2 \leq u(t) \leq 0.2, \quad t \geq 0 .
$$

Such bounds are considered in [12], but not in the earlier references [11,19,21]. Our modified optimal control problem is defined as follows: Choose u to maximize $x_{1}(T)$ subject to the dynamics (42)-(43), the stopping constraint (44), and the control bounds (49).

Using our program with $\epsilon=10$ and $\delta=10^{-2}$, the optimal results are listed in Table 2. As expected, imposing additional constraints on the angle of attack causes the final range to decrease. The optimal angle of attack functions and state trajectories are shown in Figure 5 and Figure 6. 


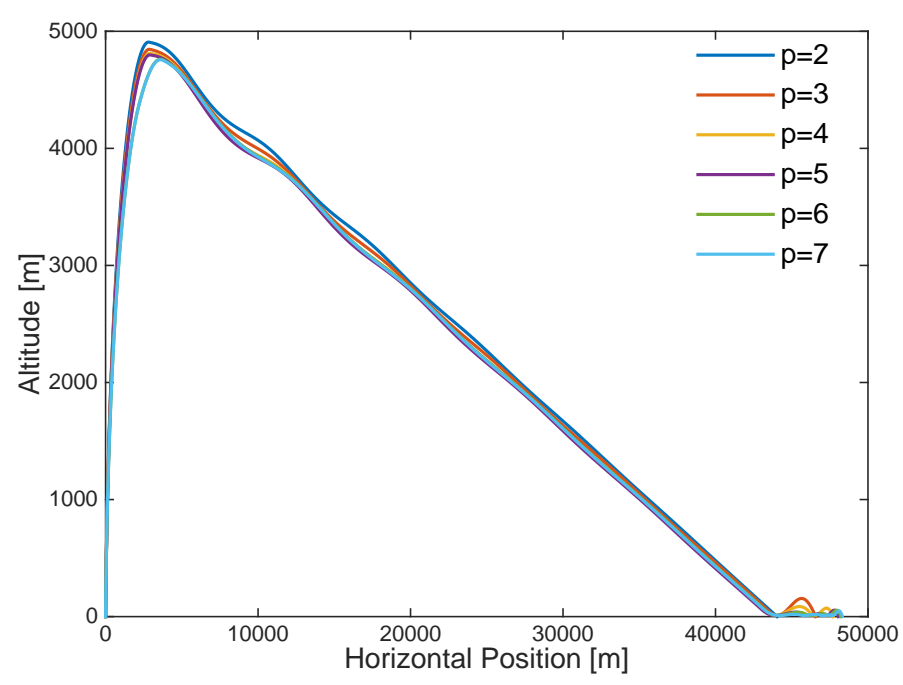

(a) Optimal flight trajectory

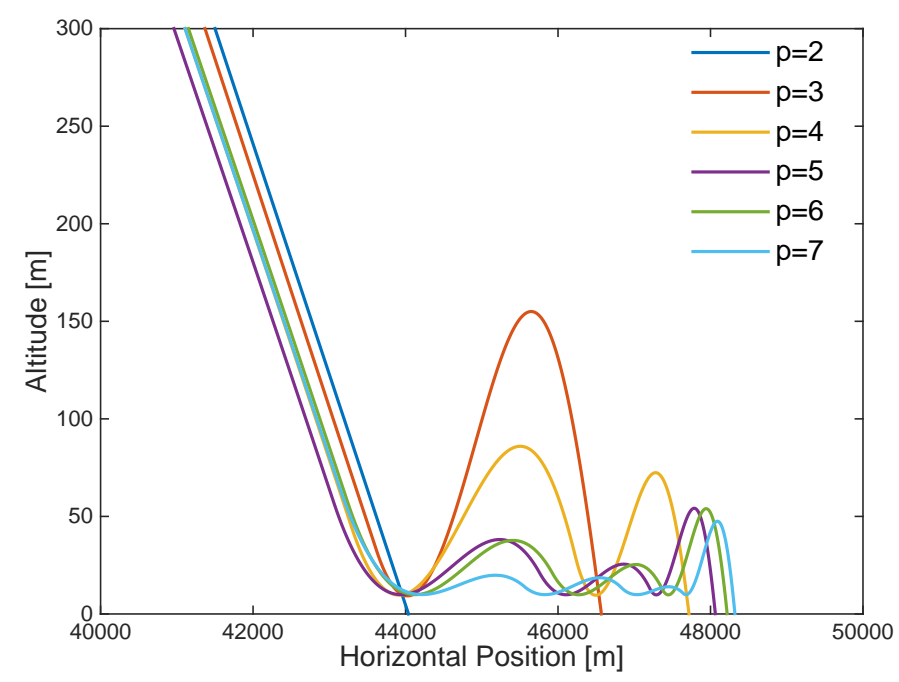

(b) Optimal flight trajectory near the terminal time

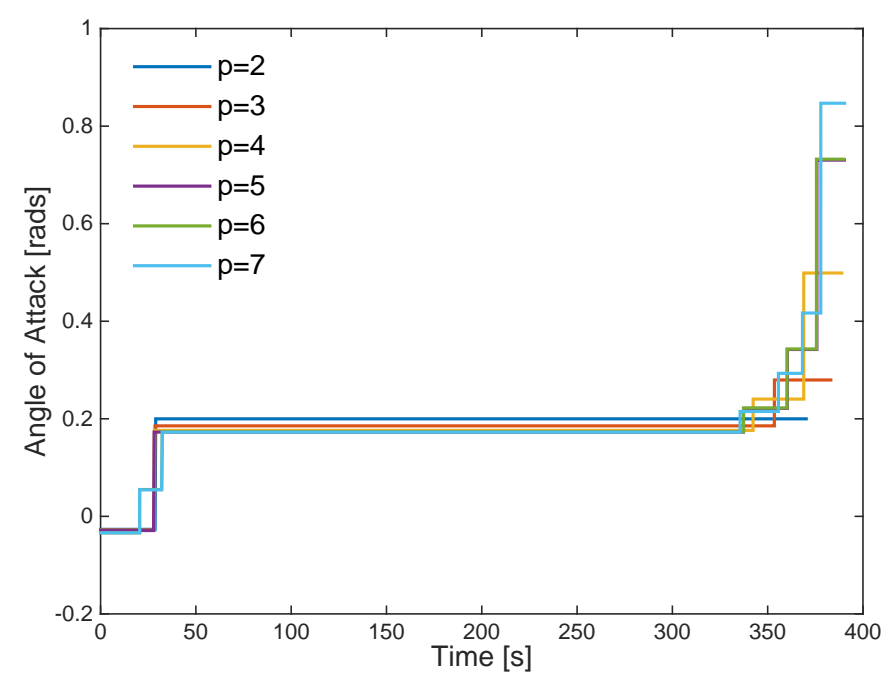

(c) Optimal angle of attack function

Fig. 2: Optimal solution of the glider control problem in Section 5.1 with piecewise-constant control. 


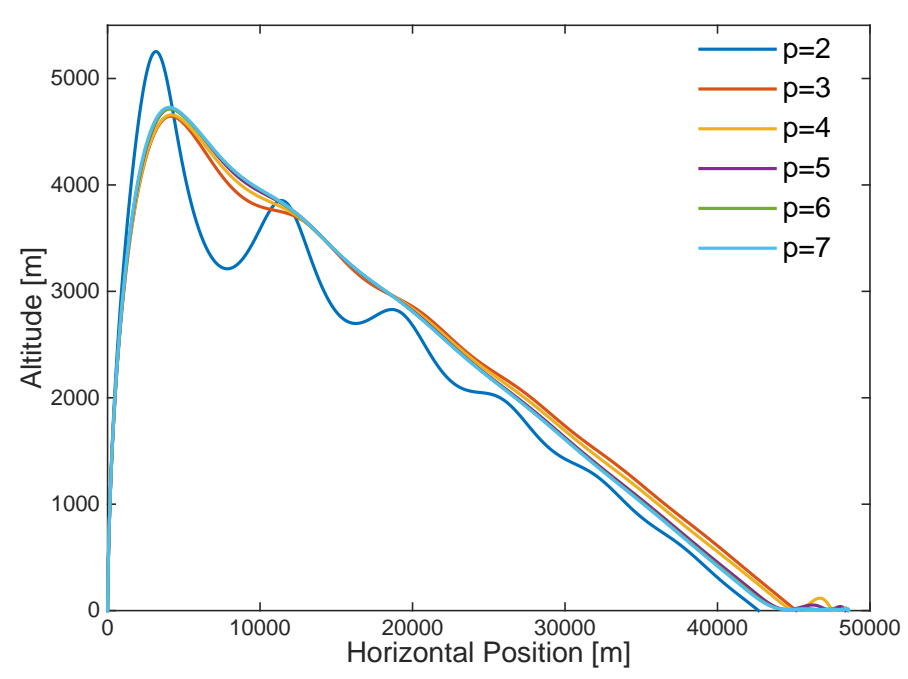

(a) Optimal flight trajectory

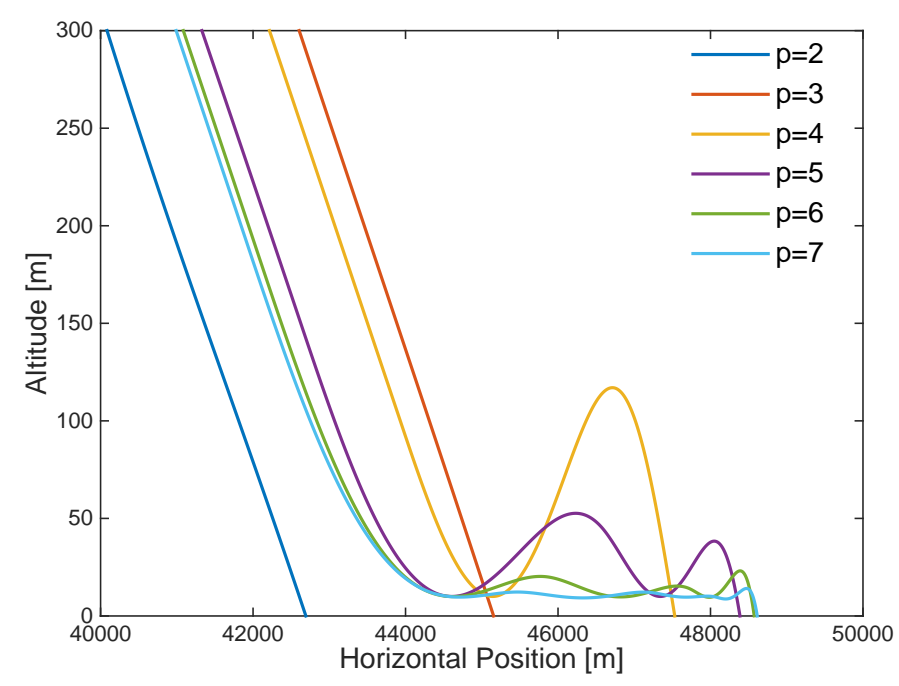

(b) Optimal flight trajectory near the terminal time

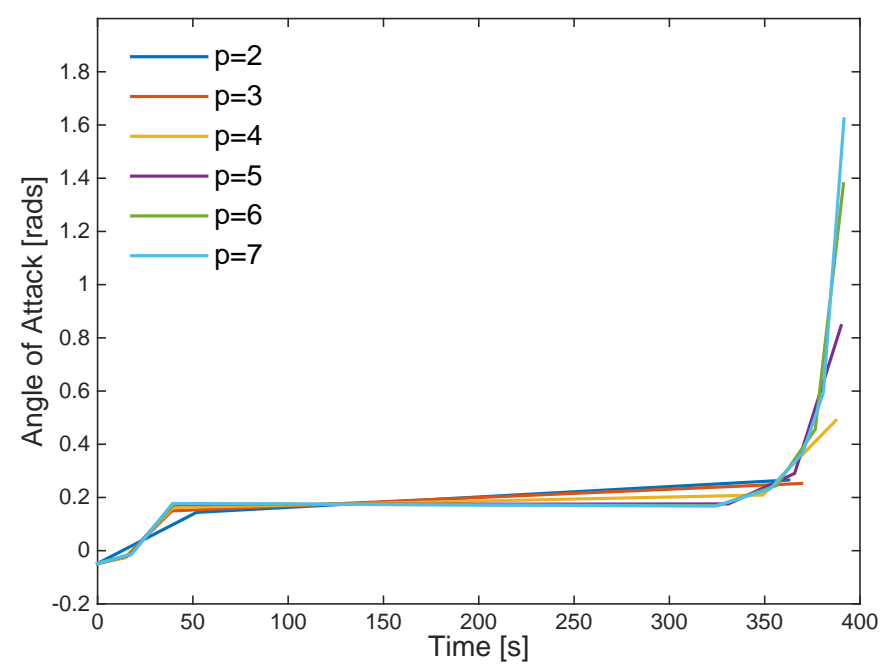

(c) Optimal angle of attack function

Fig. 3: Optimal solution of the glider control problem in Section 5.1 with piecewise-linear control. 


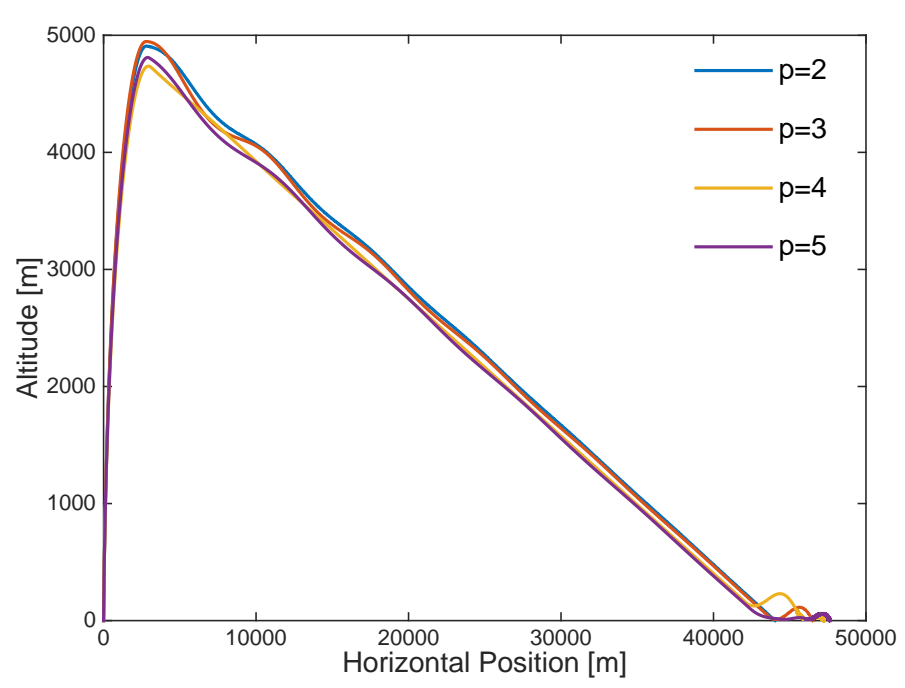

(a) Optimal flight trajectory

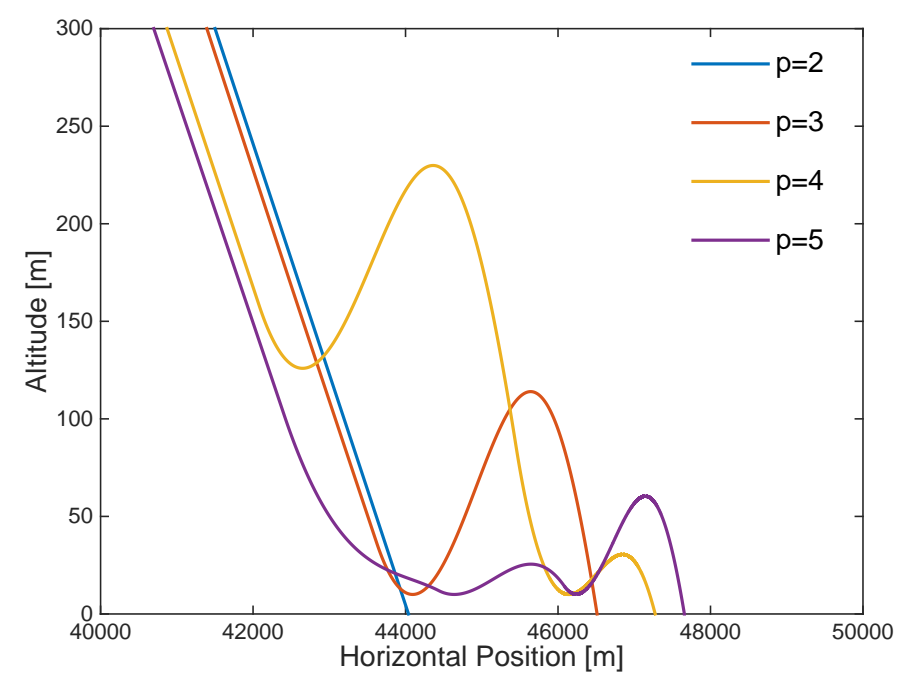

(b) Optimal flight trajectory near the terminal time

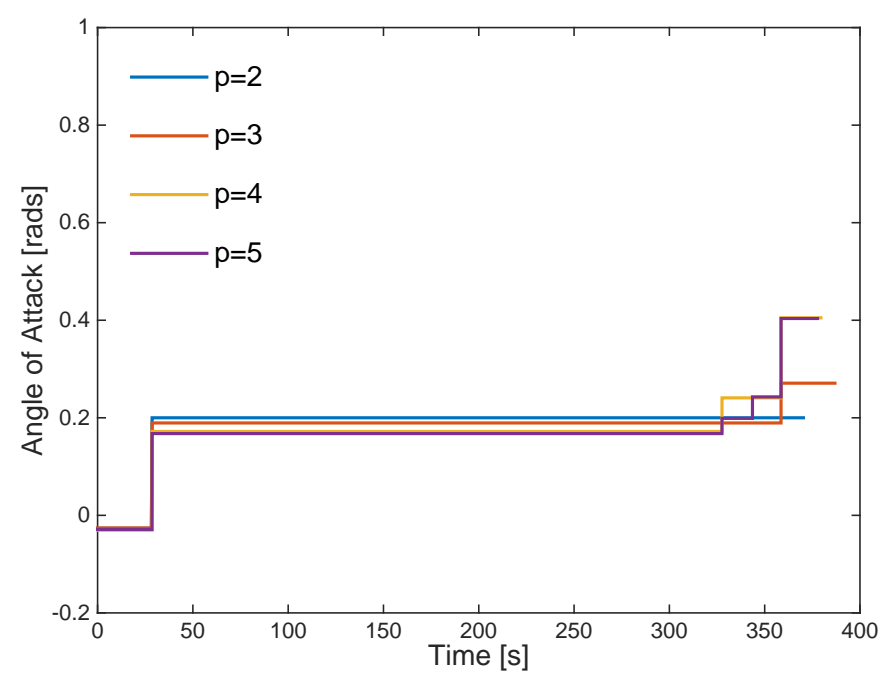

(c) Optimal angle of attack function

Fig. 4: Optimal solution of the glider control problem in Section 5.1 using the method in [12]. 


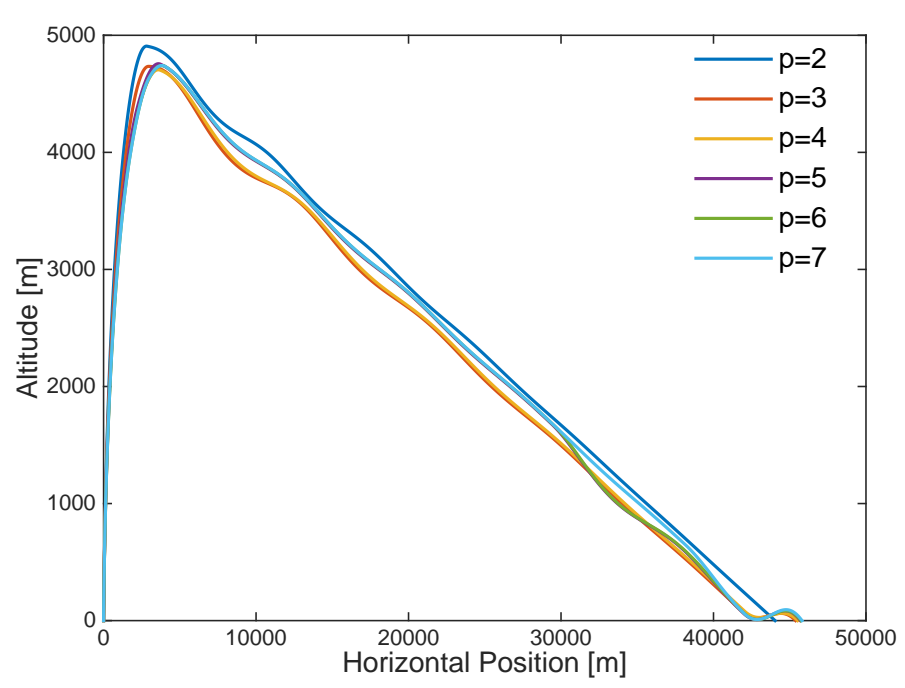

(a) Optimal flight trajectory

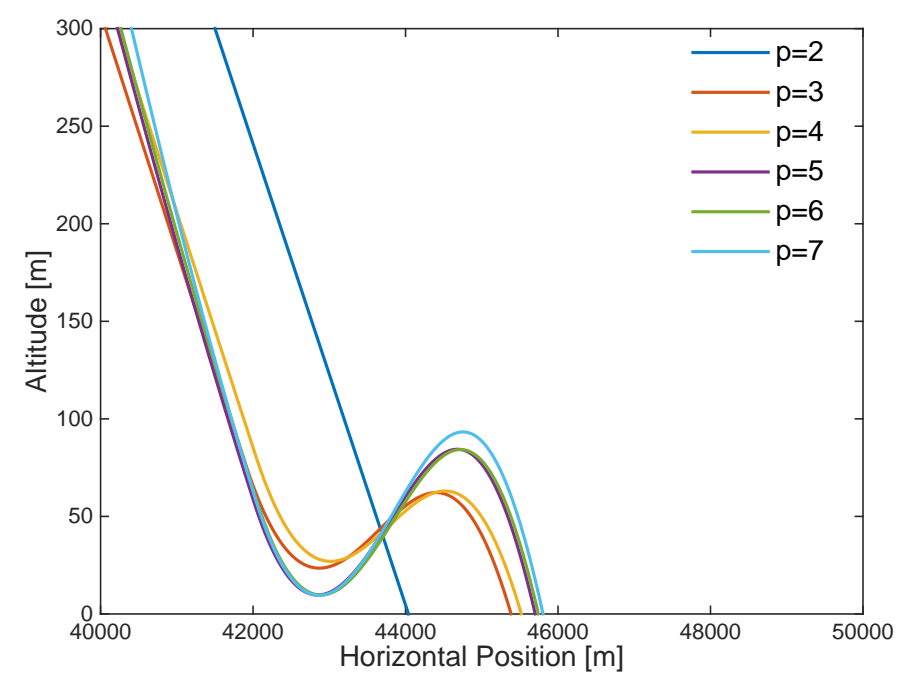

(b) Optimal flight trajectory near the terminal time

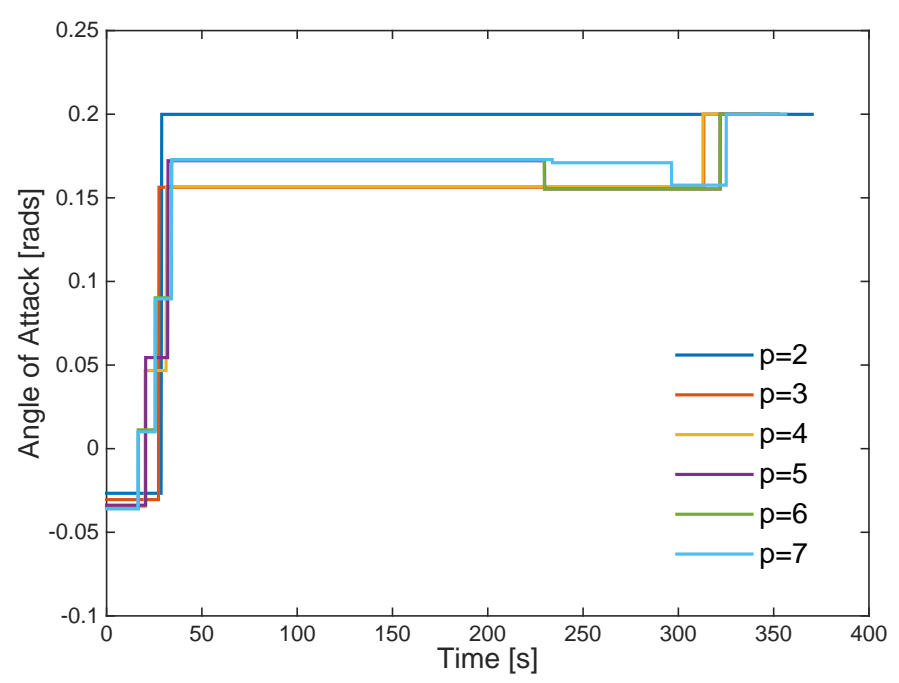

(c) Optimal angle of attack function

Fig. 5: Optimal solution of the glider control problem in Section 5.1 with piecewise-constant control and bound constraints (49). 


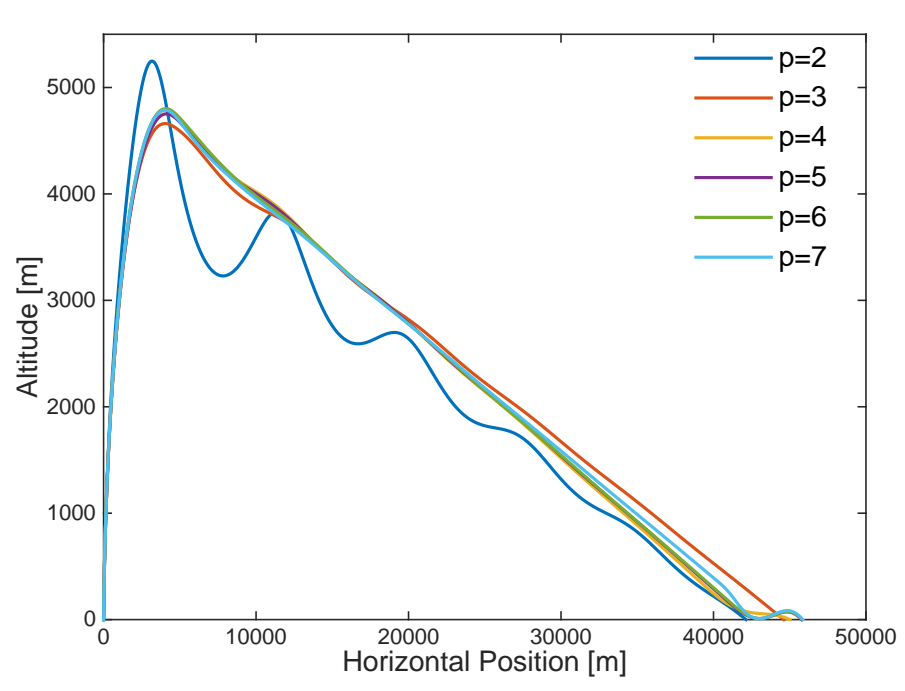

(a) Optimal flight trajectory

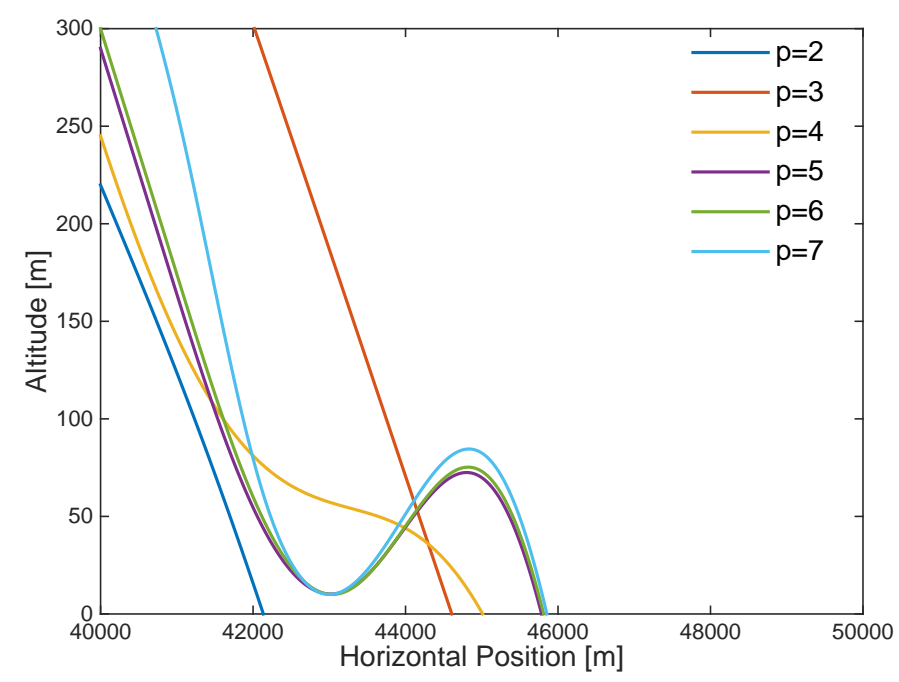

(b) Optimal flight trajectory near the terminal time

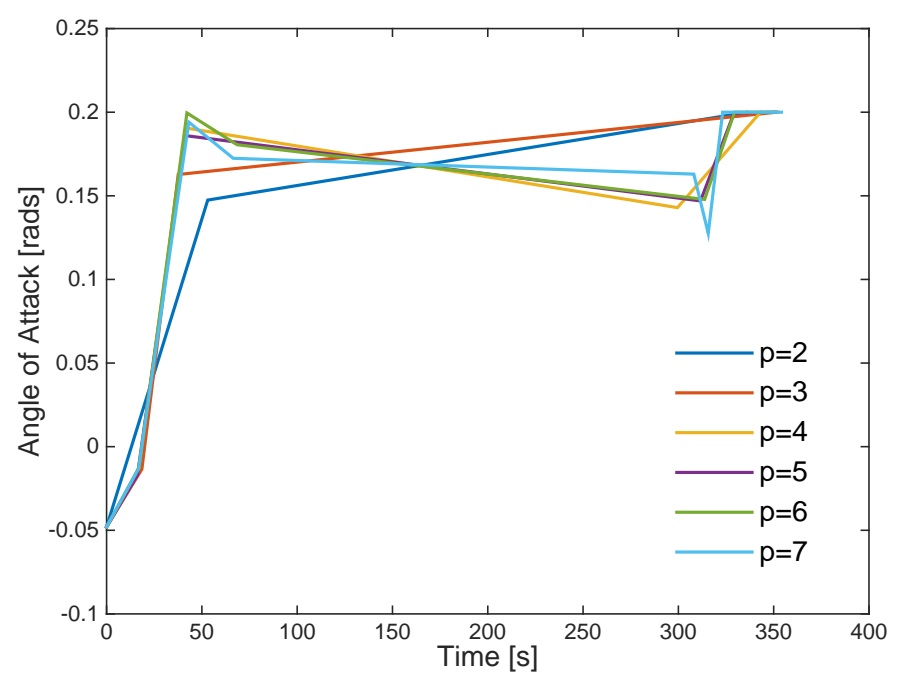

(c) Optimal angle of attack function

Fig. 6: Optimal solution of the glider control problem in Section 5.1 with piecewise-linear control and bound constraints (49). 


\subsection{Range Maximization of a Hypersonic Re-entry Vehicle}

Consider the following dynamic model for a hypersonic space vehicle re-entering the earth's atmosphere $[6,7]$ :

$$
\begin{aligned}
& \dot{x}_{1}=x_{2} \sin \left(x_{3}\right), \\
& \dot{x}_{2}=-\frac{C_{B}}{2 E^{*}} x_{2}^{2}\left(1+u^{2}\right) \exp \left(-b R x_{1}\right)-\frac{\sin \left(x_{3}\right)}{\left(x_{1}+1\right)^{2}}, \\
& \dot{x}_{3}=C_{B} x_{2} u \exp \left(-b R x_{1}\right)-\frac{\cos \left(x_{3}\right)}{x_{2}\left(x_{1}+1\right)^{2}}+\frac{x_{2} \cos \left(x_{3}\right)}{x_{1}+1}, \\
& \dot{x}_{4}=\frac{x_{2} \cos \left(x_{3}\right)}{x_{1}+1}
\end{aligned}
$$

where $x_{1}$ is the dimensionless altitude, $x_{2}$ is the dimensionless speed, $x_{3}$ is the flight path angle (in radians), $x_{4}$ is the dimensionless flight range, $u$ is the lift ratio, and $C_{B}, E^{*}, b$, and $R$ are constants.

Note that the time variable in $(50)$ is a scaled version of the original time. More specifically, the original time (in seconds) is given by $t_{d}=\sqrt{R / g} t$, where $g=9.8066352$ is the gravitational acceleration at the earth's surface. The altitude of the vehicle (in metres) is given by $R x_{1}$, the flight range (in metres) is given by $R x_{4}$, and the velocity of the vehicle (in metres per second) is given by $\sqrt{R g} x_{2}$.

The initial conditions for the state variables are

$$
x_{1}(0)=\frac{2 \times 10^{5}}{R}, \quad x_{2}(0)=\frac{7100}{\sqrt{g R}}, \quad x_{3}(0)=\frac{\pi}{36}, \quad x_{4}(0)=0 .
$$

The constants in (50)-(51) are defined as follows:

$$
C_{B}=937.639, \quad E^{*}=3.24, \quad b=1.5 \times 10^{-4}, \quad R=6.378 \times 10^{6} .
$$

We define the terminal time $T$ as the first time at which

$$
x_{1}(T)=0
$$

Furthermore, the lift coefficient is subject to the following bounds:

$$
0 \leq u(t) \leq 2, \quad t \in[0, T]
$$

We consider the problem of maximizing the range of the space vehicle subject to the following constraint on the maximum heat rate:

$$
\frac{k_{d}}{\sqrt{r_{\text {nose }}}} x_{2}(t)^{3} \exp \left(-\frac{1}{2} b R x_{1}(t)\right) \leq Q_{\max }, \quad t \in[0, T],
$$

where $r_{\text {nose }}=0.1$ is the nose radius of the vehicle, $Q_{\max }=4.5 \times 10^{3}$ is the maximum allowed heat rate, and $k_{d}=3.0748 \times 10^{4}$ is a fixed constant.

Our range maximization problem is defined as follows: Choose the control $u$ to maximize $x_{4}(T)$ subject to the dynamics (50)-(51), the stopping constraint (52), the control bounds (53), and the continuous inequality constraint (54).

This problem was formulated and solved in [6]. Full details regarding the model are given in [7]. Using our program with $\epsilon=10^{-6}$ and $\delta=10^{-3}$, the optimal results for $p=1, \ldots, 6$ are listed in Table 3 and the optimal controls and optimal state trajectories are shown in Figure 7 . The computation time for each $p=1, \ldots, 6$ took less than one minute. Using our new method with $p=6$, the maximum range can be increased from $18781.6 \mathrm{~km}$ to $21945.94 \mathrm{~km}$, and the peak heat rate can be reduced from $4501 \mathrm{~kW} / \mathrm{m}^{2}$ to $4499.9956 \mathrm{~kW} / \mathrm{m}^{2}$ compared with the method in [6]. 

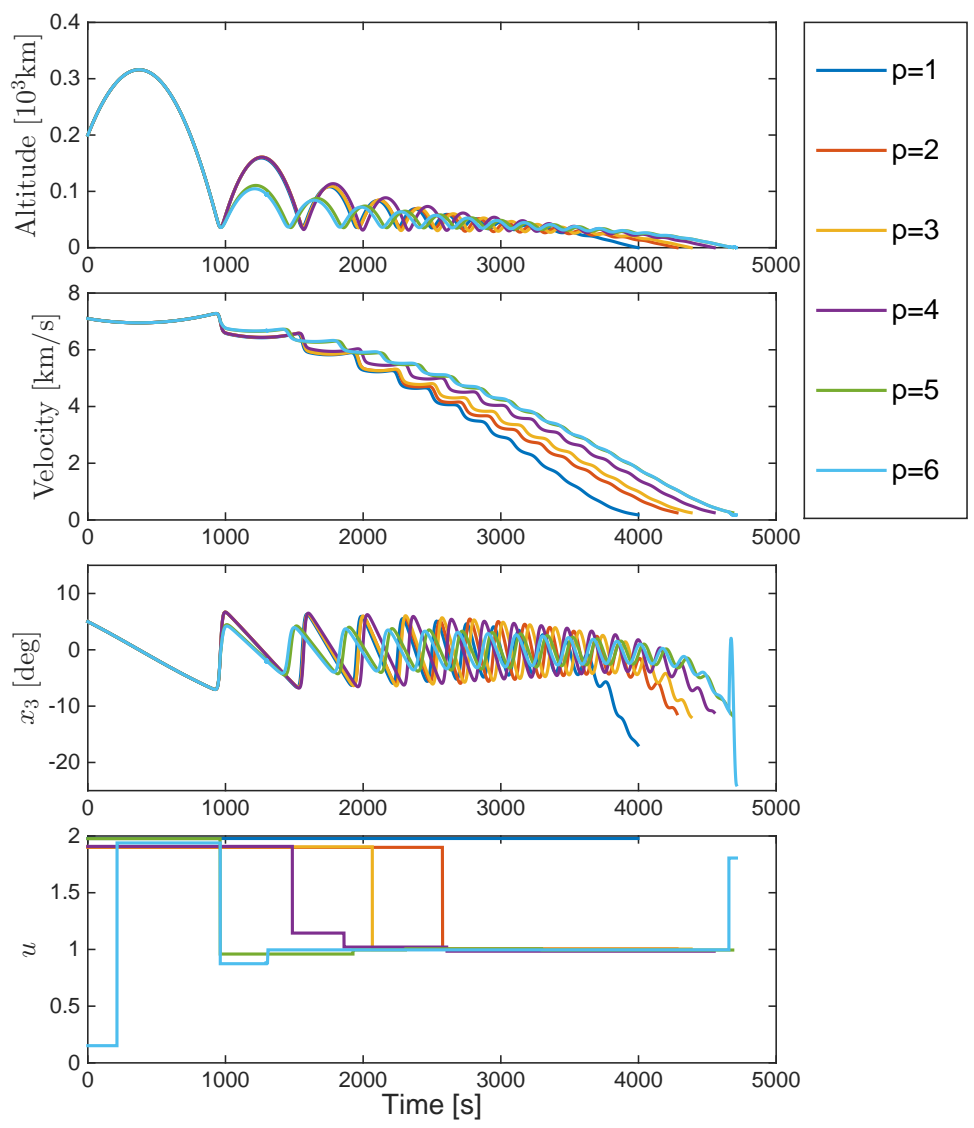

(a) Optimal flight trajectory and control

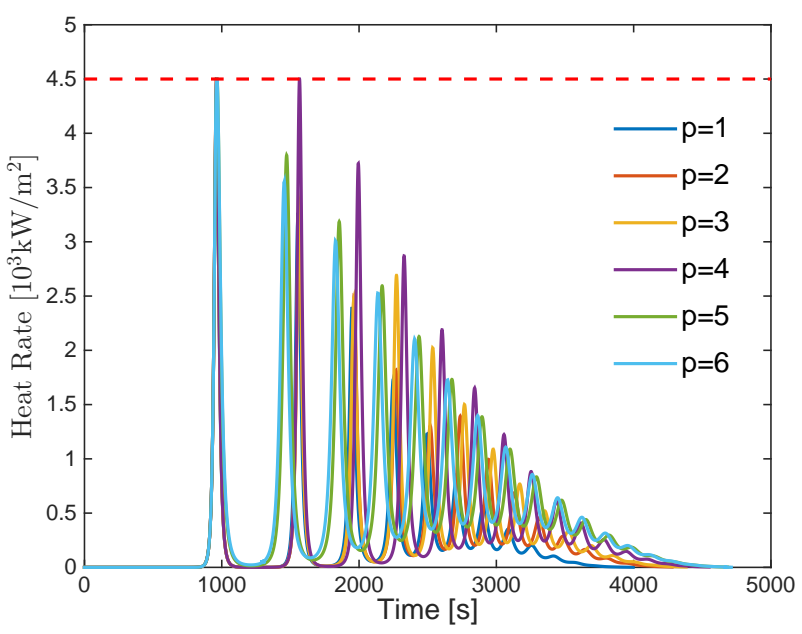

(b) Heat rate

Fig. 7: Optimal solution of the hypersonic vehicle problem in Section 5.2. 


\begin{tabular}{ccccc}
\hline$p$ & $T$ & $T_{d}[\mathrm{~s}]$ & Maximum Range $[\mathrm{km}]$ & Maximum Heat Rate $\left[\mathrm{kW} / \mathrm{m}^{2}\right]$ \\
\hline 1 & 4.96 & 3996 & 18485.65 & 4388.1352 \\
2 & 5.31 & 4280 & 19445.41 & 4500.0078 \\
3 & 5.43 & 4382 & 19928.19 & 4500.0073 \\
4 & 5.64 & 4550 & 20928.72 & 4500.0078 \\
5 & 5.81 & 4685 & 21929.77 & 4500.0075 \\
6 & 5.84 & 4711 & 21945.94 & 4499.9956 \\
\hline
\end{tabular}

Table 3: Numerical results for the hypersonic vehicle problem in Section 5.2 , where $T_{d}=T \sqrt{R / g}$. Note that the terminal altitude for each optimal trajectory is zero metres.

\subsection{Time-optimal Control of a Nuclear Reactor}

For our final example, we consider the time-optimal control of a nuclear reactor. This problem was formulated and solved in [16]. The dynamics are given by

$$
\begin{aligned}
& \dot{x}_{1}(t)=k_{1}\left(x_{3}(t)-1\right) x_{1}(t)+k_{2} x_{2}(t), \\
& \dot{x}_{2}(t)=k_{1} x_{1}(t)-k_{2} x_{2}(t), \\
& \dot{x}_{3}(t)=u(t),
\end{aligned}
$$

where $x_{1}$ is the neutron density, $x_{2}$ is the delayed neutron concentration, $x_{3}$ is the reactivity, and $k_{1}=5$ and $k_{2}=0.1$ are constants. The initial and final states are

$$
x_{1}(0)=0.04, \quad x_{2}(0)=2, \quad x_{3}(0)=0
$$

and

$$
x_{1}(T)=0.06, \quad x_{2}(T)=3, \quad x_{3}(T)=0 .
$$

The control function is subject to the following bound constraints:

$$
-0.2 \leq u(t) \leq 0.2, \quad t \in[0, T] .
$$

Our aim is to minimize the time taken to drive the system from the initial state (56) to the target state (57). This time-optimal control problem is defined as follows: Choose $u$ to minimize the terminal time $T$ subject to the dynamics (55)-(56), the terminal constraint (57), and the control bounds (58).

To convert this time-optimal control problem into the form of Problem 1, we introduce a new state variable $x_{4}$ with dynamics

$$
\dot{x}_{4}(t)=1, \quad x_{4}(0)=0 .
$$

It is clear that $x_{4}(t)=t$ for all $t \geq 0$. Thus, the time-optimal control problem defined above can be formulated in the framework of Problem 1 with

$$
\Phi(\boldsymbol{x}) \triangleq 10^{4}\left(x_{1}(T)-0.06\right)^{2}+10^{4}\left(x_{2}(T)-3\right)^{2}+10^{4} x_{3}(T)^{2}, \quad \Psi(\boldsymbol{x}) \triangleq x_{4}(T) .
$$

The stopping surface here consists of just a single point (the final state defined by (57)). Thus, only some of the admissible controls will be capable of transfering the system to the stopping surface. Consequently, the methods in $[11,12,19,21]$ for solving optimal control problems with stopping constraints are not applicable to this problem (recall that these methods are based on the assumption that the terminal time is always finite).

According to [16], the optimal control has the following bang-bang structure:

$$
u(t)= \begin{cases}0.2, & \text { if } 0 \leq t \leq \tau_{1}, \\ -0.2, & \text { if } \tau_{1} \leq t \leq \tau_{2}, \\ 0.2, & \text { if } \tau_{2} \leq t \leq T\end{cases}
$$

where $\tau_{1}$ and $\tau_{2}$ are switching times to be determined. Thus, the optimal control is a piecewiseconstant function with $p=3$ subintervals. Note that only the switching times need to be chosen optimally, not the control heights. Using our program with $\epsilon=10^{-5}, \delta=10^{-8}$, and $p=3$, we obtained a minimum terminal time of $T=7.0472$ with optimal switching times $\tau_{1}=3.3821$ and $\tau_{2}=6.9058$. The state at the terminal time is $x(T)=[0.06000344,2.99999989,-0.00000010]^{\top}$ and the optimal control and optimal states are shown in Figure 8. These results are consistent with those reported in $[16]$. 


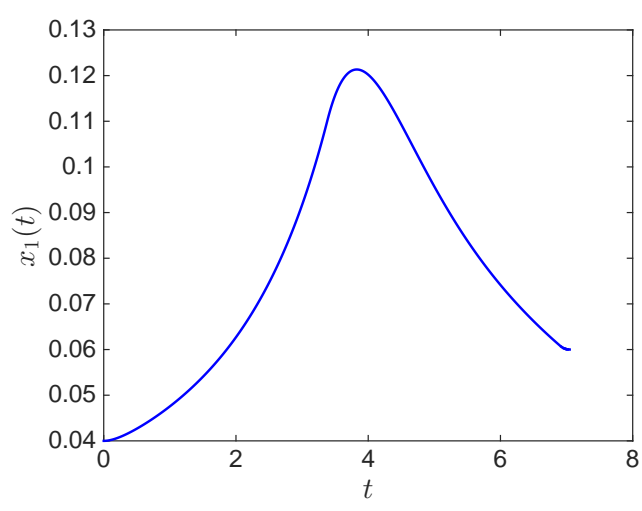

(a) State 1

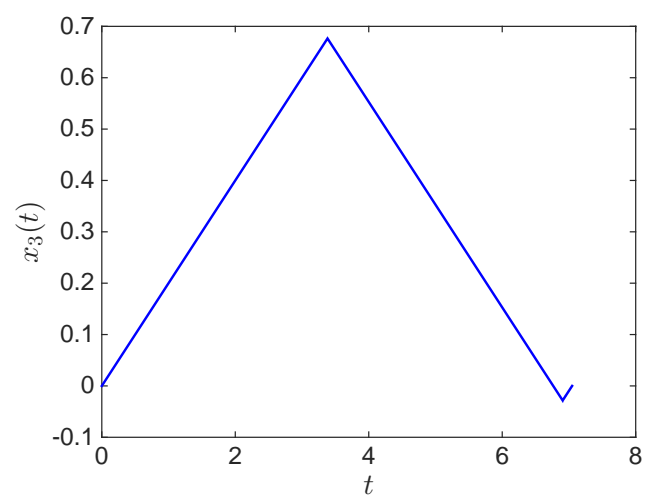

(c) State 3

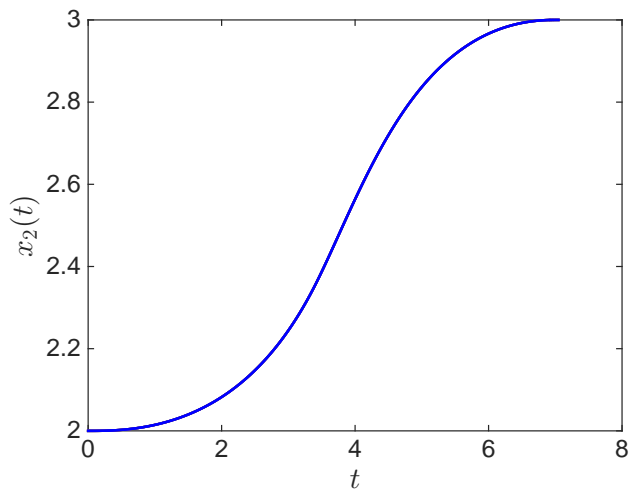

(b) State 2

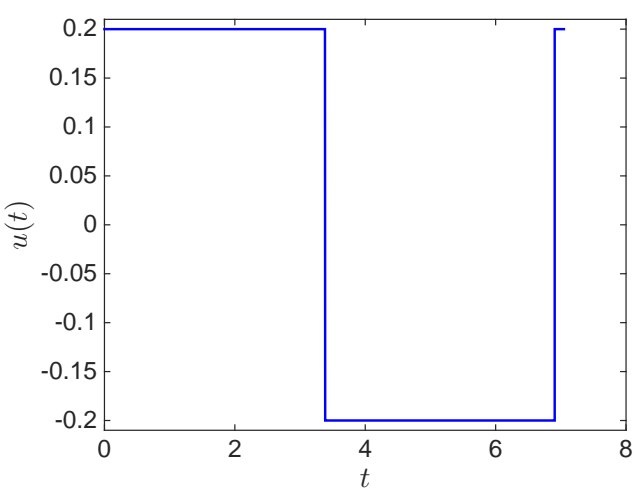

(d) Optimal control

Fig. 8: Optimal solution of the time-optimal control problem in Section 5.3.

\section{Conclusion}

This paper has introduced a novel optimal control problem in which the terminal time is a function of the control. This non-standard optimal control problem includes the classic time-optimal control problem as a special case. In terms of numerics, the main challenge with solving such problems is to ensure that the terminal time is indeed the first time at which the stopping constraint is satisfied. Conventional optimal control algorithms cannot provide this guarantee. Our main contribution is an approximation scheme - and the associated convergence analysis - for converting the non-standard optimal control problem with stopping constraints into a series of standard problems, each with fixed terminal time. Our experience with the example problems in Section 5 is that this approximation scheme is more flexible and more reliable than the existing methods in $[6,11,12,19,21]$, which rely on the restrictive assumption that the terminal time is always finite.

\section{References}

1. Ahmed, N.U.: Elements of Finite-dimensional Systems and Control Theory. Longman Scientific and Technical, Essex (1988)

2. Banihashemi, N., Kaya, C.Y.: Inexact restoration for Euler discretization of box-constrained optimal control problems. Journal of Optimization Theory and Applications 156(3), 726-760 (2013)

3. Budhiraja, A., Ross, K.: Optimal stopping and free boundary characterizations for some Brownian control problems. Annals of Applied Probability 18(6), 2367-2391 (2008)

4. Hager, W.W.: Runge-Kutta methods in optimal control and the transformed adjoint system. Numerische Mathematik 87(2), 247-282 (2000)

5. Hartl, R.F., Sethi, S.P., Vickson, R.G.: A survey of the maximum principles for optimal control problems with state constraints. SIAM Review 37(2), 181-218 (1995) 
6. Jiang, C., Lin, Q., Yu, C., Teo, K.L., Duan, G.: An exact penalty method for free terminal time optimal control problem with continuous inequality constraints. Journal of Optimization Theory and Applications 154(1), 30-53 (2012)

7. Jorris, T.R.: Common Aero Vehicle Autonomous Reentry Trajectory Optimization Satisfying Waypoint and No-fly Zone Constraints. PhD thesis, Graduate School of Engineering and Management, Air Force Institute of Technology (2007)

8. Kaya, C.Y., Martínez, J.M.: Euler discretization and inexact restoration for optimal control. Journal of Optimization Theory and Applications 134(2), 191-206 (2007)

9. Lee, H.W.J., Teo, K.L., Rehbock, V., Jennings, L.S.: Control parametrization enhancing technique for time optimal control problems. Dynamic Systems and Applications 6(2), 243-262 (1997)

10. Lin, Q., Loxton, R., Teo, K.L.: The control parameterization method for nonlinear optimal control: A survey. Journal of Industrial and Management Optimization 10(1), 275-309 (2014)

11. Lin, Q., Loxton, R., Teo, K.L., Wu, Y.H.: A new computational method for a class of free terminal time optimal control problems. Pacific Journal of Optimization 7(1), 63-81 (2011)

12. Lin, Q., Loxton, R., Teo, K.L., Wu, Y.H.: Optimal control computation for nonlinear systems with statedependent stopping criteria. Automatica 48(9), 2116-2129 (2012)

13. Lin, Q., Loxton, R., Teo, K.L., Wu, Y.H., Yu, C.: A new exact penalty method for semi-infinite programming problems. Journal of Computational and Applied Mathematics 261(1), 271-286 (2014)

14. Loxton, R., Teo, K.L., Rehbock, V., Yiu, K.F.C.: Optimal control problems with a continuous inequality constraint on the state and the control. Automatica 45(10), 2250-2257 (2009)

15. Luenberger, D.G., Ye, Y.: Linear and Nonlinear Programming. 3rd edition. Springer, New York (2008)

16. Maurer, H., Osmolovskii, N.P.: Second order sufficient conditions for time-optimal bang-bang control. SIAM Journal on Control and Optimization 42(6), 2239-2263 (2004)

17. Nocedal, J., Wright, S.J.: Numerical Optimization. 2nd edition. Springer, New York (2006)

18. Schittkowski, K.: NLPQLP: A Fortran Implementation of a Sequential Quadratic Programming Algorithm with Distributed and Non-Monotone Line Search. University of Bayreuth, Bayreuth (2007)

19. Teo, K.L., Goh, C.J., Lim, C.C.: A computational method for a class of dynamical optimization problems in which the terminal time is conditionally free. IMA Journal of Mathematical Control and Information 6(1), 81-95 (1989)

20. Teo, K.L., Goh, C.J., Wong, K.H.: A Unified Computational Approach to Optimal Control Problems. Longman Scientific and Technical, Essex (1991)

21. Teo, K.L., Jepps, G., Moore, E.J., Hayes, S.: A computational method for free time optimal control problems, with application to maximizing the range of an aircraft-like projectile. Journal of the Australian Mathematical Society - Series B 28(3), 393-413 (1987)

22. Zhang, M., Sun, Y., Duan, G., Wang, G.: Reentry trajectory optimization of hypersonic vehicle with minimum heat. In Proceedings of the 8th World Congress on Intelligent Control and Automation, Jinan, China (2010)

23. Zhao, Y., Stadtherr, M.A.: Rigorous global optimization for dynamic systems subject to path constraints. Industrial and Engineering Chemistry Research 50(22), 12678-12693 (2011) 